\title{
DUAL PROCESSES TO SOLVE SINGLE SERVER SYSTEMS August 2004
}

\author{
Alan Krinik ${ }^{1}$, Gerardo Rubino ${ }^{2}$, Daniel Marcus ${ }^{1}$, Randall J. Swift ${ }^{1}$, Hassan Kasfy $^{1}$, Holly Lam ${ }^{1}$
}

\begin{abstract}
$\underline{\text { Abstract }}$
A new approach is used to determine the transient probability functions of the classical queueing systems: $\mathrm{M} / \mathrm{M} / 1, \mathrm{M} / \mathrm{M} / 1 / \mathrm{H}$, and $\mathrm{M} / \mathrm{M} / 1 / \mathrm{H}$ with catastrophes. This new solution method uses dual processes, randomization and lattice path combinatorics. The method reveals that the transient probability functions for $\mathrm{M} / \mathrm{M} / 1 / \mathrm{H}$ and $\mathrm{M} / \mathrm{M} / 1 / \mathrm{H}$ with catastrophes have the same mathematical form.
\end{abstract}

Mathematics Subject Classifications: $\quad$ 60K25, 60J27.

Keywords: Dual processes; Single server queueing system; Transient probability functions; $\mathrm{M} / \mathrm{M} / 1$; Lattice path combinatorics; Randomization; M/M/1/H; Catastrophes

\section{Introduction}

For over fifty years, determining new methods to obtain the transient probability functions of the classical single server queueing systems has captured and maintained the interest of theorists and practitioners alike. In this article, a new sample path approach that combines dual processes, randomization and lattice path combinatorics is used to obtain the transient probability functions of three single server queueing systems.

Section 2 contains background material and some important results connecting the transition probability functions of a birth-death process and its dual process. The transient probability functions of the classical $\mathrm{M} / \mathrm{M} / 1$ queueing system are re-derived using dual processes and the reflection principle in section 3. In section 4, the transient probability functions of the $\mathrm{M} / \mathrm{M} / 1 / \mathrm{H}$ system are determined and formulated in terms of dual processes. The solution method again relies upon lattice path combinatorics instead of the traditional eigenvalue approach. Section 4 also contains an interesting formula for counting the number of lattice paths going from state $j$ to state $k$ in $n$ steps confined within a given horizontal strip.

The dual process approach generalizes to certain non birth-death processes. In section 5, the transient probability functions of an $\mathrm{M} / \mathrm{M} / 1 / \mathrm{H}$ system with catastrophes is determined. The analysis is surprisingly related to a suitably modified solution of the $\mathrm{M} / \mathrm{M} / 1 / \mathrm{H}$ system as described in section 4 . In this way, the dual process/randomization/lattice path combinatorics approach may unify the complicated analysis of finding transient probability functions for well known Markovian queueing systems.

\section{Dual Processes}

Consider a general birth-death process having transition birth rates $\lambda_{i}$ for $i=0,1,2, \ldots$ and transition death rates $\mu_{i}$ for $i=1,2,3, \ldots$ as shown in the state rate transition diagram, Figure 1. All of these rates are assumed to be nonnegative numbers. The state space may be finite or countable.

\footnotetext{
${ }^{1}$ Department of Mathematics, California State Polytechnic University, Pomona, CA 91768, USA

2 INRIA, IRISA, Campus Universitaire de Beaulieu, 35042 RENNES Cedex, France
} 


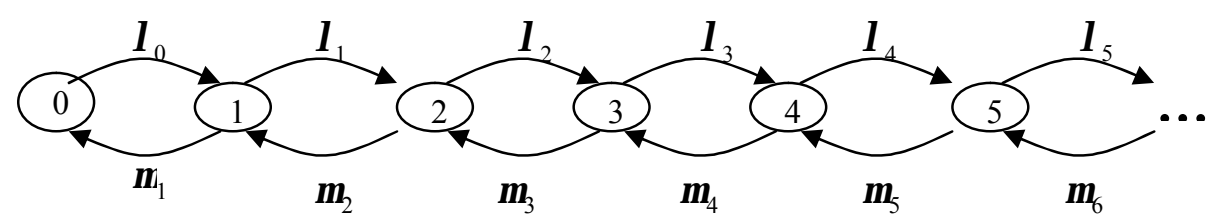

Figure 1

Our interest is to determine $P_{i, j}(t)$, the transient probability functions where $i, j=0,1,2,3, \ldots$. For a finite or countable state space with uniformly bounded transition rates, $P_{i, j}(t)$ is determined by solving the Kolmogorov backward or forward equations, see Bhattacharya and Waymire (1990) or Gross and Harris (1985). The result is a system of differential equations that may be written in matrix form as

$$
P^{\prime}(t)=Q \cdot P(t)=P(t) \cdot Q
$$

where

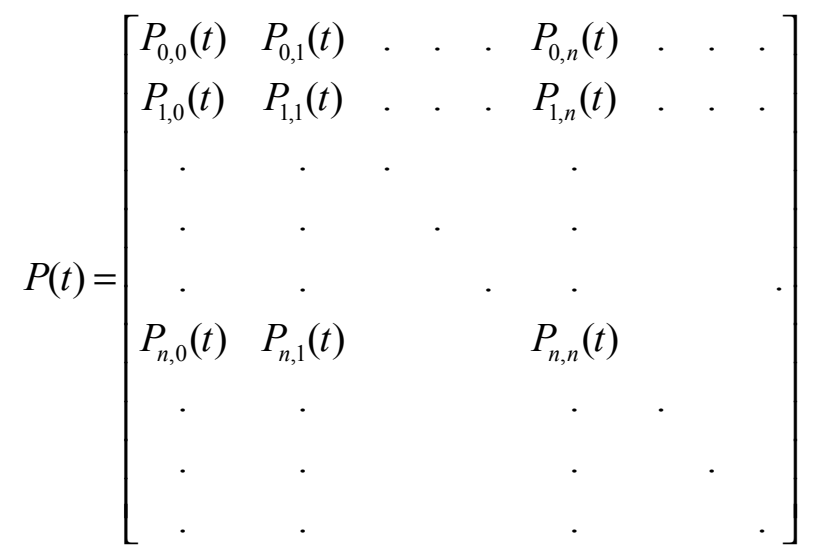

is the matrix of transition probability functions and

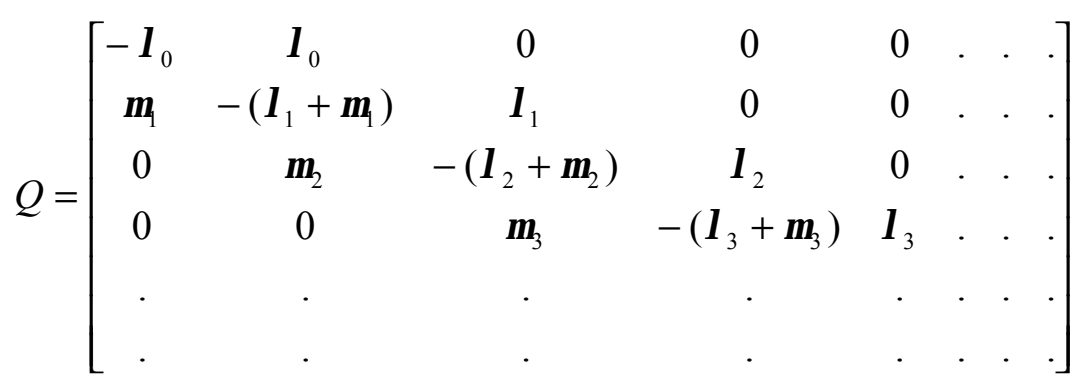

is the transition rate matrix. In the finite dimensional case, the matrix form of the backward equation is well posed. However, in the infinite dimensional case, we assume throughout this 
article that the rates $\lambda_{i}, i=0,1,2, \ldots$ and $\mu_{i}, i=1,2,3, \ldots$ are uniformly bounded. This guarantees that the birth-death system has a unique transition probability solution (cf. Anderson (1991)). The solution of the Kolmogorov backward equation may then be written, see Bhattacharya and Waymire (1990), as

$$
P(t)=e^{Q t}
$$

The dual process of the general birth-death process of Figure 1 has a state rate transition diagram as shown in Figure 2.

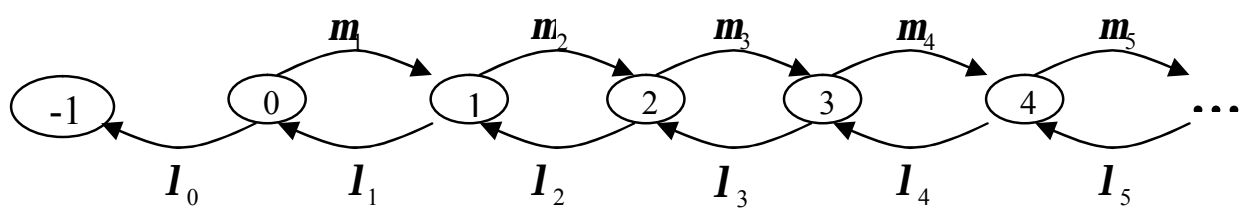

Figure 2

The transient probability functions of this dual process are denoted by $P_{i, j}^{*}(t)$. By Proposition 2.3 on page 269 of Anderson (1991), the following relationship holds between a general birthdeath system and its dual.

Theorem 1. If $P_{i, j}(t)$ and $P_{i, j}^{*}(t)$ are the transient probability functions of the Markov processes corresponding to Figure 1 and 2 respectively, then

$$
P_{i, j}(t)=\sum_{k=i}^{\infty}\left[P_{j, k}^{*}(t)-P_{j-1, k}^{*}(t)\right] \quad \text { and } \quad P_{i, j}^{*}(t)=\sum_{k=0}^{i}\left[P_{j, k}(t)-P_{j+1, k}(t)\right]
$$

for all states $i, j=0,1,2,3 \ldots$ with the convention $P_{-1, k}^{*}(t)=0$ if $\mathrm{k}>-1$.

The proof of this result is nicely presented in Anderson (1991) and depends upon the forward and backward equations and some algebraic simplifications. Consequently, if the transient probability functions in either the original birth-death process or dual system are known, then the transient probability functions in the other system are as well.

Corollary 1. If $P_{i, j}(t)$ and $P_{i, j}^{*}(t)$ are the transient probability functions corresponding to the Markov processes depicted in Figures 1 and 2 respectively, then

$$
P_{i, j}(t)=\sum_{k=-1}^{i-1}\left[P_{j-1, k}^{*}(t)-P_{j, k}^{*}(t)\right]
$$

for all states $i, j=0,1,2,3 \ldots$ with the convention $P_{-1, k}^{*}(t)=0$ if $\mathrm{k}>-1$. 
For the general birth-death process (Figure 1), a Markov chain called the associated randomized chain (Figure 3) is considered.

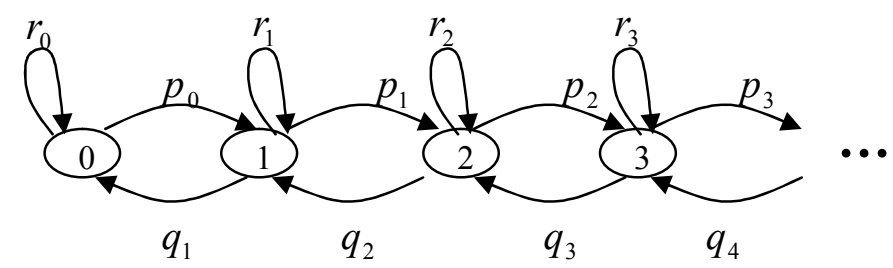

Figure 3

where $r_{0}=1-\frac{\lambda_{0}}{b}, p_{i}=\frac{\lambda_{i}}{b}$ for $i=0,1,2,3, \ldots$ and $q_{i}=\frac{\mu_{i}}{b}, r_{i}=1-\frac{\lambda_{i}+\mu_{i}}{b}$ provided that $i=1,2,3, \ldots$ with $b$ chosen such that $\sup \left|\lambda_{i}+\mu_{i}\right| \leq b<\infty$ and by convention, $\mu_{0}=0$. The following theorem, called randomization (or uniformization), is a well-known result used primarily for the numerical computation of the transition probability functions $P_{i, j}(t)$ of a Markov process, (cf., Gross and Harris (1985). It applies for the preceding birth death processes of Figure 1 and, more generally, for any Markov process (with countable state space) having uniformly bounded diagonal transition rates in the $Q$ matrix.

Theorem 2. (Randomization) Suppose a Markov process (on a countable state space) has transition rate matrix $Q$ with $\sup _{i \geq 0}\left|q_{i, i}\right| \leq b<\infty$ then the transition probability functions, $P_{i, j}(t)$, may be written as

$$
P_{i, j}(t)=e^{-b t} \sum_{n=0}^{\infty} \frac{(b t)^{n}}{n !} P_{i, j}^{(n)} \text { for } i, j=0,1,2,3, \ldots
$$

where $P_{i, j}^{(n)}$ is the $n$-step transition probability of the associated randomized Markov chain.

It should be noted that $P_{i, j}(t)$ is completely determined once $P_{i, j}^{(n)}$ is "known". For examples of solving for $P_{i, j}(t)$ by finding $P_{i, j}^{(n)}$, see Bohm et al. (1997), Chang et al. (2004), Green et al. (2003), Krinik et al. (1997) and Leguesdron et al. (1993).

It is also necessary to randomize the dual process in Figure 2, obtaining

$$
P_{i, j}^{*}(t)=e^{-b t} \sum_{n=0}^{\infty} \frac{(b t)^{n}}{n !} P_{i, j}^{*(n)}
$$


where $P_{i, j}^{*(n)}$ is the $n$-step transition probability of the Markov Chain shown in the diagram below.

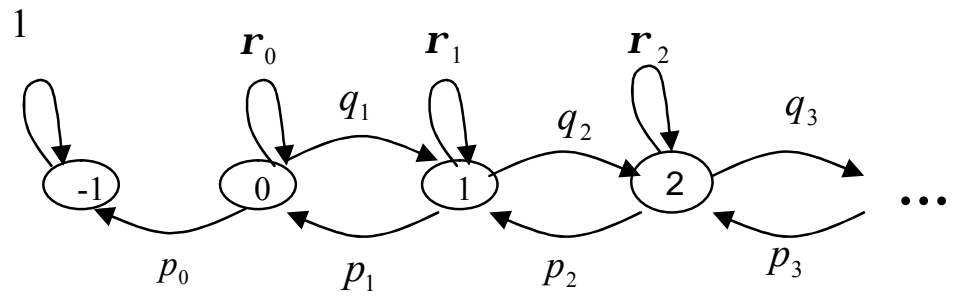

Figure 4

where $p_{i}=\frac{\lambda_{i}}{b}, q_{i}=\frac{\mu_{i}}{b}, \rho_{i}=1-p_{i}-q_{i+1}$ for $i=0,1,2,3, \ldots$ and $b$ is greater than or equal to the supremum of the absolute value of the diagonal rates of $Q^{*}$. Note the assumption that the transition rates in $Q$ are uniformly bounded implies a common value, $b$, for the randomizations of the processes, appearing in both Figures 1 and 2, can be found.

\section{The M/M/1 Queueing System}

We now consider the classical single server queueing system, M/M/1, seen in Figure 5. The birth (or arrival) rates and the death (or service) rates are positive constants represented by $\lambda$ and $\mu$ respectively. The state space of natural numbers represents the number of customers in the queue at any time $t . P_{i, j}(t)$ represents the transient probability functions, for $i, j=0,1,2,3, \ldots$ in the following state rate transition diagram.

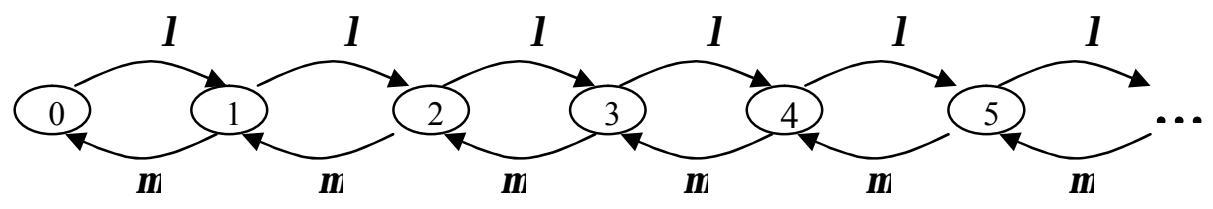

Figure 5

The associated randomized Markov chain is shown in the following state transition probability diagram.

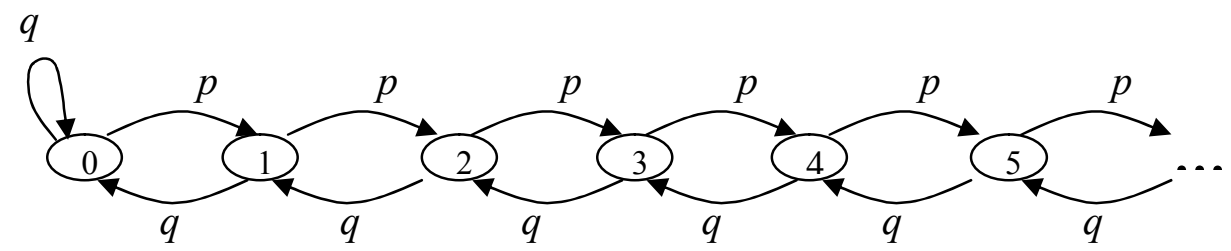

Figure 6 
with $p=\frac{\lambda}{\lambda+\mu}$ and $q=\frac{\mu}{\lambda+\mu}$. Suppose $P_{i, j}^{*}(t)$ are the transient probability functions of the dual $\mathrm{M} / \mathrm{M} / 1$ process

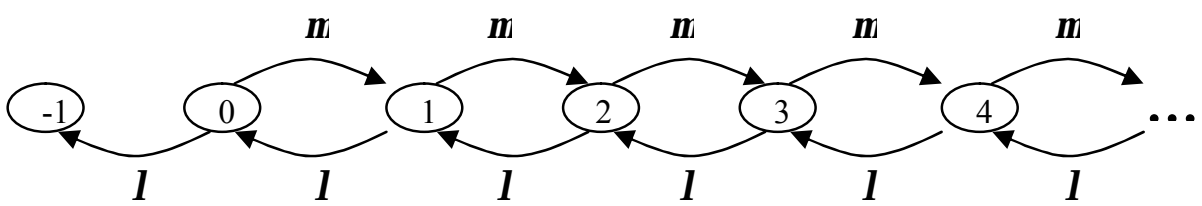

Figure 7

The randomized Markov chain of Figure 7 is shown in Figure 8.

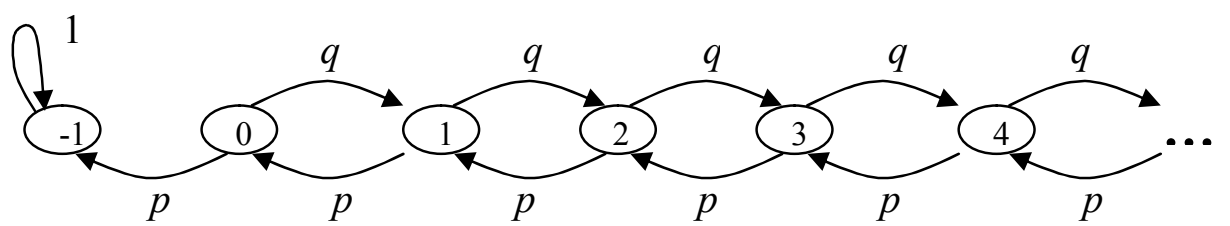

Figure 8

By Randomization, with $b=\lambda+\mu$, the transition probability functions are

$$
P_{j, k}^{*}(t)=e^{-(\lambda+\mu) t} \sum_{n=0}^{\infty} \frac{[(\lambda+\mu) t]^{n}}{n !} P_{j, k}^{*(n)}
$$

In order to obtain the transient probabilities for the $M / M / 1$ system, we calculate $P_{j, k}^{*(n)}$ for $j, k \geq 0$. The advantage of the dual process approach is the facility in counting sample paths in Figure 8 as compared to sample paths in Figure 6, see Krinik et al. (1997). To find $P_{j, k}^{*(n)}$, the sample paths in Figure 8 going from state $j$ to state $k$ in $n$-steps need to be counted. These sample paths may be visualized, for example, as a lattice path (Figure 9), where it is assumed here that $n \geq|k-j|$.

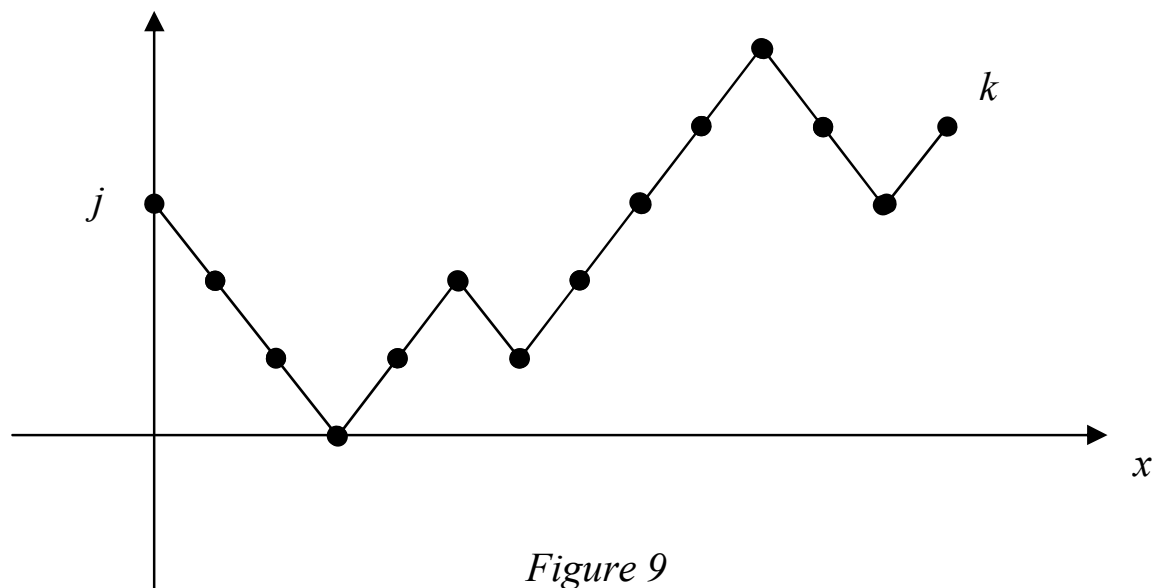


Note that $u+d=n$ and $u-d=k-j$ where $u$ is the number of upward steps and $d$ is the number of downward steps. Solving for $u$ and $d$ gives

$$
u=\frac{n+k-j}{2} \text { and } d=\frac{n-k+j}{2}
$$

so

$$
P_{j, k}^{*(n)}=(\text { number of sample paths from } j \text { to } k \text { in } n \text {-steps }) \cdot q^{u} p^{d} \text {. }
$$

To count the number of sample paths going from $j$ to $k$ in Figure 8 in $n$-steps, the associated lattice paths are counted. However, some lattice paths are not realizable as a sample path as seen in Figure 8. For example, the black lattice path from $j$ to $k$ drawn in Figure 10 is such a path.

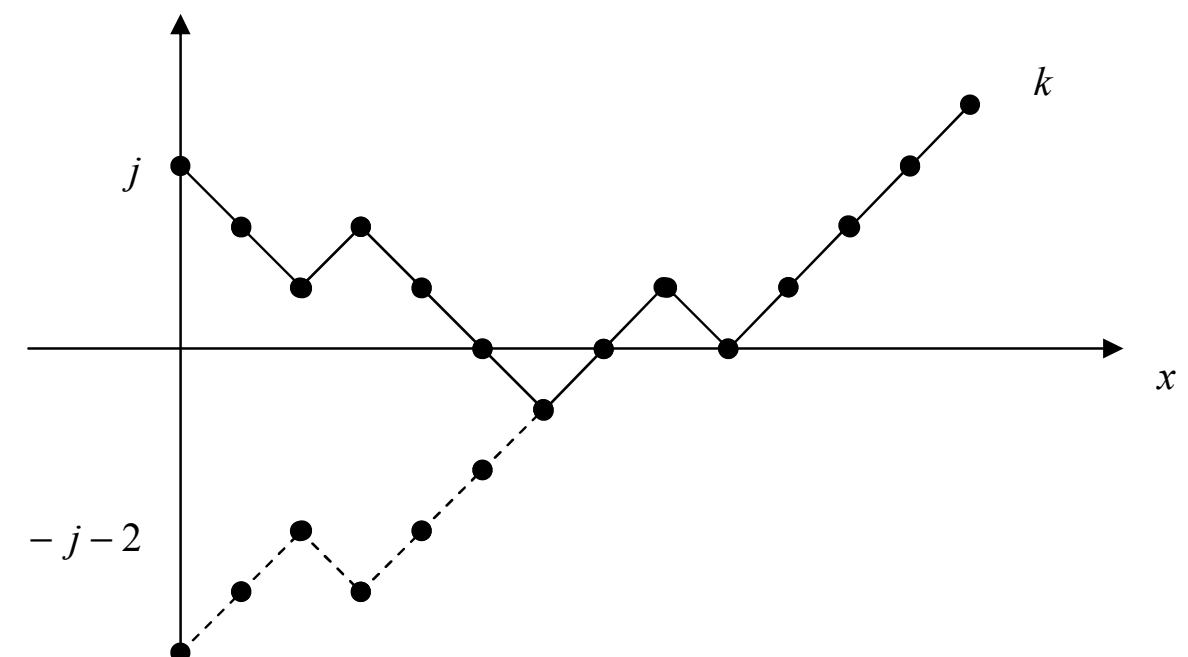

Figure 10

Fortunately, these types of lattice paths may be counted by means of a one-to-one correspondence with lattice paths going from starting point $-j-2$ to destination $k$ in $n$-steps (shown in Figure 10 as the dashed/black lattice path). This is the well known reflection principle, see Mohanty (1979) or Narayana (1979) which leads to

(number of sample paths in Figure 8 from $j$ to $k$ in $n$-steps) $=\left[\left(\begin{array}{c}n \\ \frac{n+k-j}{2}\end{array}\right)-\left(\begin{array}{c}n \\ \frac{n+k+j+2}{2}\end{array}\right)\right]$. 
Dual Processes To Solve Single Server Systems

Therefore

$$
P_{j, k}^{*(n)}=\left[\left(\begin{array}{c}
n \\
\frac{n+k-j}{2}
\end{array}\right)-\left(\begin{array}{c}
n \\
\frac{n+k+j+2}{2}
\end{array}\right)\right] q^{\frac{n+k-j}{2}} p^{\frac{n-k+j}{2}}
$$

for $j, k \geq 0$. Note this expression equals 0 when $n<|k-j|$ using the usual conventions concerning binomial coefficients. By Theorem 2 (Randomization),

$$
P_{j, k}^{*}(t)=e^{-(\lambda+\mu) t} \sum_{n=0}^{\infty} \frac{[(\lambda+\mu) t]^{n}}{n !}\left[\left(\begin{array}{c}
n \\
\frac{n+k-j}{2}
\end{array}\right)-\left(\begin{array}{c}
n \\
\frac{n+k+j+2}{2}
\end{array}\right)\right] q^{\frac{n+k-j}{2} p^{\frac{n-k+j}{2}}}
$$

and by Theorem 1 ,

$$
\begin{gathered}
P_{i, j}(t)=\sum_{k=i}^{\infty}\left[P_{j, k}^{*}(t)-P_{j-1, k}^{*}(t)\right] \\
=e^{-(\lambda+\mu) t} \sum_{k=i n=0}^{\infty} \sum_{n=\{}^{\infty}\left[\left\{[(\lambda+\mu) t]^{n}\left[\left(\begin{array}{c}
n \\
n !
\end{array}\right]-\left(\begin{array}{c}
n \\
\frac{n+k-j}{2}
\end{array}\right)-\left(\frac{n+k+j+2}{2}\right)\right] q^{\frac{n+k-j}{2} p \frac{n-k+j}{2}}\right\}-\right. \\
\left\{\frac{[(\lambda+\mu) t]^{n}}{n !}\left[\left(\begin{array}{c}
n \\
\frac{n+k-j+1}{2}
\end{array}\right)-\left(\frac{n+k+j+1}{2}\right)\right] \frac{n+k-j+1}{2} \frac{n-k+j-1}{2}\right\}
\end{gathered}
$$

for $i=0,1,2, \ldots$ and $j=1,2, \ldots$ and

$$
\begin{gathered}
P_{i, 0}(t)=\sum_{k=i}^{\infty}\left[P_{0, k}^{*}(t)-P_{-1, k}^{*}(t)\right] \\
=e^{-(\lambda+\mu) t} \sum_{k=i}^{\infty} \sum_{n=0}^{\infty}\left[\left\{\frac { [ ( \lambda + \mu ) t ] ^ { n } } { n ! } \left[\left(\begin{array}{c}
n \\
n+k \\
2
\end{array}\right)-\left(\begin{array}{c}
n \\
\left.\left.\left.\frac{n+k+2}{2}\right)\right] q^{\frac{n+k}{2}} p^{\frac{n-k}{2}}\right\}
\end{array}\right.\right.\right.\right.
\end{gathered}
$$

for $i=0,1,2, \ldots$. The preceding expressions are explicit solutions of the transient probability functions, $P_{i, j}(t)$, for the classical $\mathrm{M} / \mathrm{M} / 1$ queueing system. These results are equivalent to expressions appearing in Bohm et al. (1997), Krinik et al. (1997) and Leguesdron et al. (1993); see Lam (2004) for verification of this equivalence. The dual process with 
randomization approach provides a simplification of the analysis presented in Krinik et al. (1997) and Leguesdron et al. (1993). Note the preceding expressions may be written in randomized form as

$$
P_{i, j}(t)=e^{-(\lambda+\mu) t} \sum_{n=0}^{\infty} \frac{[(\lambda+\mu) t]^{n}}{n !} P_{i, j}^{(n)}
$$

where

$$
\begin{aligned}
& P_{i, j}^{(n)}= \\
& \underset{k=i}{n+j}\left\{\left[\left(\begin{array}{c}
n \\
\frac{n+k-j}{2}
\end{array}\right)-\left(\begin{array}{c}
n \\
\frac{n+k+j+2}{2}
\end{array}\right)\right] q^{\frac{n+k-j}{2}} p^{\frac{n-k+j}{2}}-\left[\left(\begin{array}{c}
n \\
\frac{n+k-j+1}{2}
\end{array}\right)-\left(\begin{array}{c}
n \\
\frac{n+k+j+1}{2}
\end{array}\right)\right] \begin{array}{cc}
\frac{n+k-j+1}{2} & \frac{n-k+j-1}{2} \\
q &
\end{array}\right\}= \\
& (\sqrt{q p})^{n}\left(\sqrt{\frac{p}{q}}\right)^{j} \sum_{k=i}^{n+j}\left(\sqrt{\frac{q}{p}}\right)^{k}\left\{\left[\left(\begin{array}{c}
n \\
\frac{n+k-j}{2}
\end{array}\right)\left(\begin{array}{c}
n \\
\frac{n+k+j+2}{2}
\end{array}\right)\right]-\sqrt{\frac{q}{p}}\left[\left(\begin{array}{c}
n \\
\frac{n+k-j+1}{2}
\end{array}\right)\left(\begin{array}{c}
n \\
\frac{n+k+j+1}{2}
\end{array}\right)\right]\right\}= \\
& \left(\frac{\sqrt{\lambda \mu}}{\lambda+\mu}\right)^{n}\left(\sqrt{\frac{\lambda}{\mu}}\right)^{j} \sum_{k=i}^{n+j}\left(\sqrt{\frac{\mu}{\lambda}}\right)^{k}\left\{\left[\left(\begin{array}{c}
n \\
\frac{n+k-j}{2}
\end{array}\right)\left(\begin{array}{c}
n \\
\frac{n+k+j+2}{2}
\end{array}\right)\right]-\sqrt{\frac{\mu}{\lambda}}\left[\left(\begin{array}{c}
n \\
\frac{n+k-j+1}{2}
\end{array}\right)-\left(\begin{array}{c}
n \\
\frac{n+k+j+1}{2}
\end{array}\right)\right]\right\}
\end{aligned}
$$

where $P_{i, j}^{(n)}$ is the $n$-step transition probability from $i$ to $j$ on Figure 6 where $i=0,1,2, \ldots$ and $j=1,2, \ldots$. Note by our conventions, two of the binomial coefficients within this summand vanish for any given $n$ and $k$. And

$$
P_{i, 0}^{(n)}=\sum_{k=i}^{n}\left[\left(\begin{array}{c}
n \\
\frac{n+k}{2}
\end{array}\right)-\left(\begin{array}{c}
n \\
\frac{n+k+2}{2}
\end{array}\right)\right] q^{\frac{n+k}{2}} p^{\frac{n-k}{2}}=\left(\frac{\sqrt{\lambda \mu}}{\lambda+\mu}\right)^{n} \sum_{k=i}^{n}\left(\sqrt{\frac{\mu}{\lambda}}\right)^{k}\left[\left(\begin{array}{c}
n \\
\frac{n+k}{2}
\end{array}\right)-\left(\begin{array}{c}
n \\
\frac{n+k+2}{2}
\end{array}\right)\right]
$$

where $P_{i, 0}^{(n)}$ is the $n$-step transition probability from $i$ to 0 on Figure 6 where $i=0,1,2, \ldots$.

Alternative expressions for $P_{i, j}(t), P_{i, 0}(t), P_{i, j}^{(n)}, P_{i, 0}^{(n)}$ in terms of a finite summation over $k$ from -1 to $i-1$ may be found by using Corollary 1 in place of Theorem 1 . Details of this alternative development for M/M/1 may be found in Lam (2004). The Corollary 1 approach is also demonstrated in the next section to find the transient probability functions of the $\mathrm{M} / \mathrm{M} / 1 / \mathrm{H}$ queueing system. 


\section{Dual Processes To Solve Single Server Systems}

\section{The $M / M / 1 / H$ Queueing System}

We now consider the classical single server queueing system having finite capacity $H$, $\mathrm{M} / \mathrm{M} / 1 / \mathrm{H}$, seen in Figure 11. The birth (or arrival) rates and the death (or service) rates are positive constants represented by $\lambda$ and $\mu$ respectively. The state space $\{0,1,2, \ldots, H\}$ represents the number of customers in the queue at any time $t . P_{i, j}(t)$ represents the transient probability functions, for $i, j=0,1,2,3, \ldots, H$ in the following state rate transition diagram.

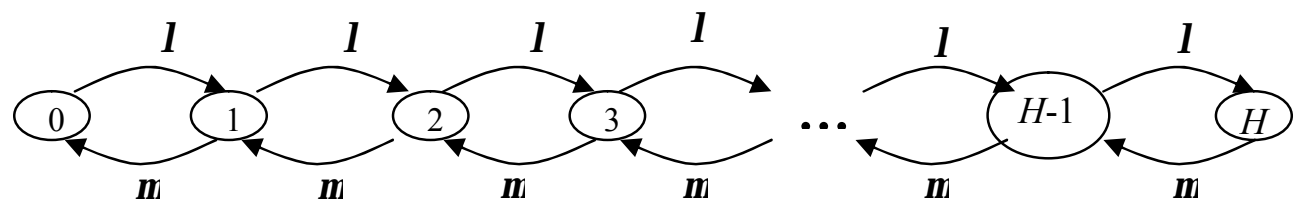

Figure 11

The associated randomized Markov chain is shown in the following state transition probability diagram.

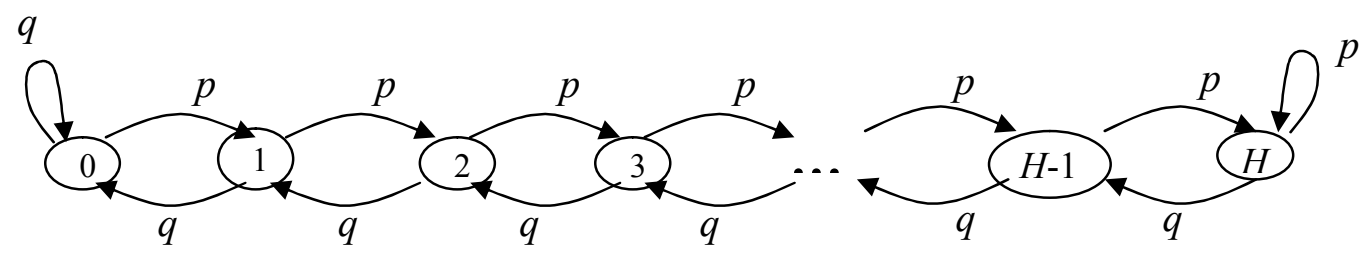

Figure 12

with $p=\frac{\lambda}{\lambda+\mu}$ and $q=\frac{\mu}{\lambda+\mu}$.

Let $P_{i, j}^{*}(t)$ denote the transient probability functions of the dual $\mathrm{M} / \mathrm{M} / 1 / \mathrm{H}$ process having transition rate diagram in Figure 13.

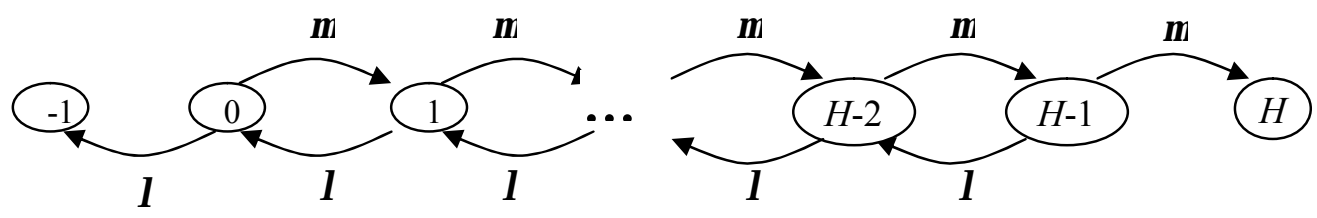

Figure 13

The randomized Markov chain of the process depicted in Figure 13 is shown in Figure 14.

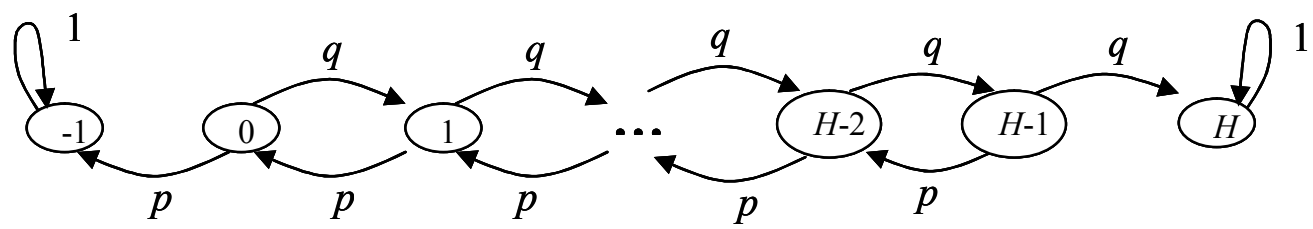

Figure 14 
By randomization, with $b=\lambda+\mu$, the transition probability functions are

$$
P_{j, k}^{*}(t)=e^{-(\lambda+\mu) t} \sum_{n=0}^{\infty} \frac{[(\lambda+\mu) t]^{n}}{n !} P_{j, k}^{*(n)}
$$

In order to obtain the transient probabilities for the $\mathrm{M} / \mathrm{M} / 1 / \mathrm{H}$ system using Corollary 1 of section 2, we again need to calculate $P_{j, k}^{*(n)}$. For now, assume $j, k$ are restricted to the following set of states $\{0,1,2, \ldots, H-1\}$. Consider the set of sample paths, $L_{j, k}^{(n)}(H)$, going from state $j$ to state $k$ in $n$-steps in Figure 14. These sample paths may be visualized as lattice paths confined to a horizontal strip bounded by lines $y=-1$ and $y=H$ as shown in Figure 15. The paths never touch either of these boundaries. In order to determine $P_{j, k}^{*(n)}$, we wish to count the number of paths in $L_{j, k}^{(n)}(H)$.

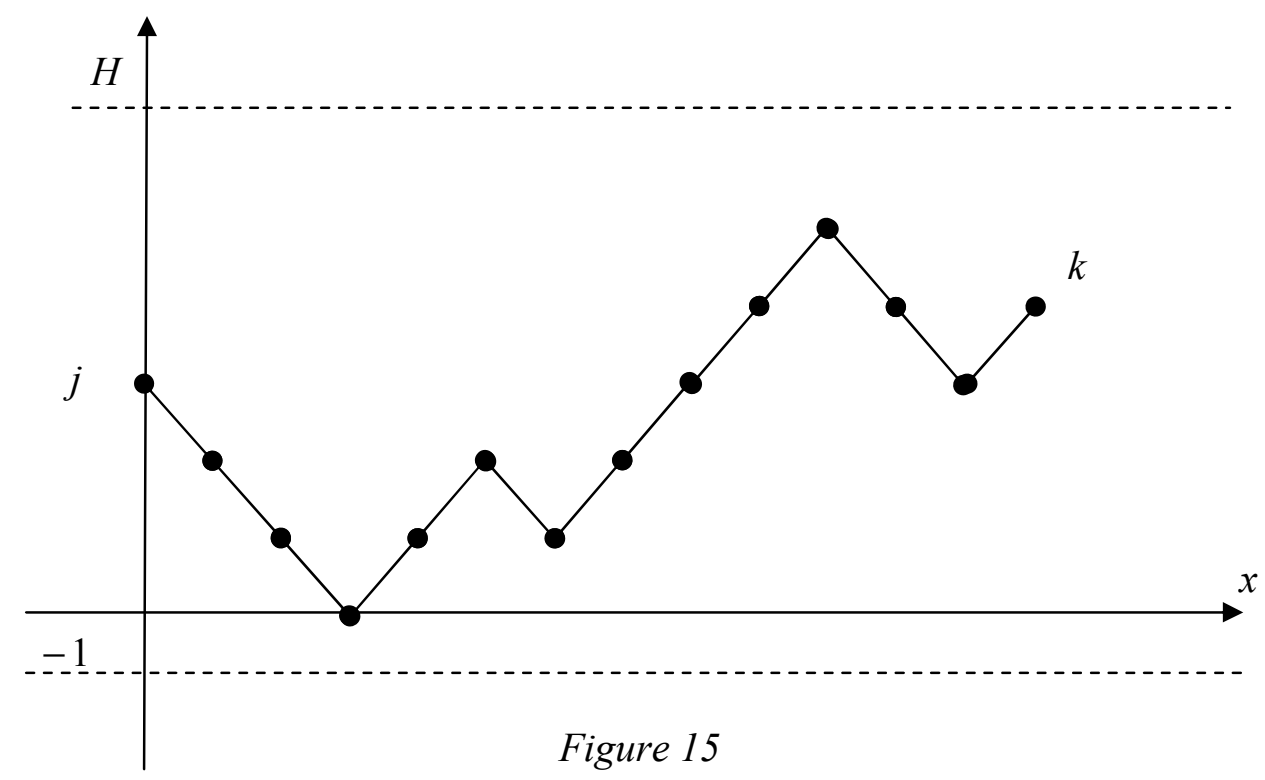

As in section $3, u+d=n$ and $u-d=k-j$ where $u$ is the number of upward steps and $d$ is the number of downward steps. Solving for $u$ and $d$ gives

$$
u=\frac{n+k-j}{2} \text { and } d=\frac{n-k+j}{2}
$$

so

$$
P_{j, k}^{*(n)}=\left|L_{j, k}^{(n)}(H)\right| q^{u} p^{d}
$$




\section{Dual Processes To Solve Single Server Systems}

where $\left|L_{j, k}^{(n)}(H)\right|$ denotes the number of paths in $L_{j, k}^{(n)}(H)$. To count the number of sample paths in $L_{j, k}^{(n)}(H)$, we use the following generalization of the reflection principle which may be found in Mohanty (1979) and Narayana (1979).

$$
\left|L_{j, k}^{(n)}(H)\right|=\sum_{l}\left\{\left(\begin{array}{c}
n \\
\frac{n-k+j}{2}-l(H+1)
\end{array}\right)_{+}-\left(\begin{array}{c}
n \\
\frac{n+k+j}{2}+l(H+1)+1
\end{array}\right)\right\}_{+}
$$

where $l$ takes all integer values positive, negative and zero and $\left(\begin{array}{l}y \\ z\end{array}\right)_{+}=\left(\begin{array}{l}y \\ z\end{array}\right)$ when $\mathrm{y} \geq \mathrm{z}$ and $\left(\begin{array}{l}y \\ z\end{array}\right)_{+}=0$ when $z<0$ or $y<z$. We recast this result using the following lemma.

\section{Lemma 1.}

$$
\begin{aligned}
\sum_{g \equiv a \bmod m}\left(\begin{array}{l}
n \\
g
\end{array}\right)_{+} & =\frac{1}{m}\left[\omega^{-a}(1+\omega)^{n}+\omega^{-2 a}\left(1+\omega^{2}\right)^{n}+\omega^{-3 a}\left(1+\omega^{3}\right)^{n}+\ldots+\omega^{-m a}\left(1+\omega^{m}\right)^{n}\right] \\
& =\frac{1}{m} \sum_{u=1}^{m} \omega^{-u a}\left(1+\omega^{u}\right)^{n}
\end{aligned}
$$

where $a<m, m$ and $a$ are positive integers and $\omega=\exp \left\{\frac{2 \pi i}{m}\right\}$ is the $m^{\text {th }}$ root of unity.

\section{Proof.}

Apply the binomial theorem $(x+y)^{n}=\sum_{g=0}^{n}\left(\begin{array}{l}n \\ g\end{array}\right) x^{g} y^{n-g} \quad m$-times. First let $x=\omega$ and $y=1$, then let $x=\omega^{2}$ and $y=1$, then let $x=\omega^{3}$ and $y=1$, etc. until $x=\omega^{m}$ and $y=1$. So we have and $\left(\omega^{u}+1\right)^{n}=\sum_{g=0}^{n}\left(\begin{array}{l}n \\ g\end{array}\right)\left(\omega^{u}\right)^{g} 1^{n-g}$ for $u=1,2, \ldots m$. Multiply both sides of this equation by $\omega^{-u a}$ and sum over $u=1, \ldots, m$ getting

$$
\sum_{u=1}^{m} \omega^{-u a}\left(\omega^{u}+1\right)^{n}=\sum_{u=1}^{m} \sum_{g=0}^{n}\left(\begin{array}{l}
n \\
g
\end{array}\right) \omega^{u(g-a)}=\sum_{g=0}^{n}\left(\begin{array}{l}
n \\
g
\end{array}\right) \sum_{u=1}^{m} \omega(g-a)
$$


The result follows by realizing that $\sum_{u=1}^{m} \omega^{u(g-a)}=m$ when $g \equiv a \bmod m$ because $\omega$ is the $m^{\text {th }}$ root of unity and $\sum_{u=1}^{m} \omega^{u(g-a)}=0$ when $g \neq a \bmod m$ which again is a consequence of $\omega$ being the $m^{\text {th }}$ root of unity. This completes the proof of the lemma.

Apply Lemma 1 with $m=H+1$ and $a=a_{1}=\frac{n-k+j}{2}$ and $g=\frac{n-k+j}{2}-l(H+1)$ and $\omega=\exp \left\{\frac{2 \pi i}{H+1}\right\}$ to obtain

$$
\sum_{l}\left(\frac{n-k+j}{2}-l(H+1)\right)_{+}=\frac{1}{m} \sum_{u=1}^{m} \omega^{-u a_{1}}\left(1+\omega^{u}\right)^{n}
$$

Similarly,

$$
\sum_{l}\left(\frac{n+k+j}{2}+l(H+1)+1\right)_{+}^{n}=\frac{1}{m} \sum_{u=1}^{m} \omega^{-u a_{2}}\left(1+\omega^{u}\right)^{n}
$$

follows from Lemma 1 when $m=H+1, a=a_{2}=\frac{n+k+j}{2}+1$ and $g=\frac{n+k+j}{2}+l(H+1)+1$ and $\omega=\exp \left\{\frac{2 \pi i}{H+1}\right\}$. Therefore

$$
\begin{aligned}
\left|L_{j, k}^{(n)}(H)\right| & =\sum_{l}\left\{\left(\frac{n-k+j}{2}-l(H+1)\right)_{+}-\left(\frac{n+k+j}{2}+l(H+1)+1\right)\right\}_{+} \\
& =\frac{1}{H+1} \sum_{u=1}^{H+1} \omega^{-u a_{1}}\left(1+\omega^{u}\right)^{n}-\frac{1}{H+1} \sum_{u=1}^{H+1} \omega^{-u a_{2}\left(1+\omega^{u}\right)^{n} \quad \text { by Lemma } 1 .} \\
& =\frac{1}{H+1} \sum_{u=1}^{H+1}\left(\omega^{\left.-u a_{1}-\omega^{-u a_{2}}\right)\left(1+\omega^{u}\right)^{n}}\right. \\
& =\frac{1}{H+1} \sum_{u=1}^{H+1}\left(\omega^{-u\left(\frac{n-k+j}{2}\right)}-\omega^{-u\left(\frac{n+k+j}{2}\right)}\right)\left(1+\omega^{u}\right)^{n} \text { by substitution. }
\end{aligned}
$$




$$
\begin{aligned}
& =\frac{1}{H+1} \sum_{u=1}^{H+1}\left(\omega^{-u\left(\frac{n+j}{2}\right)} \omega^{\frac{u k}{2}}-\omega^{-u\left(\frac{n+j}{2}\right)} \omega^{\frac{-u k}{2}} \omega^{-u}\right)\left(1+\omega^{u}\right)^{n} \\
& =\frac{1}{H+1} \sum_{u=1}^{H+1}\left(\omega^{\frac{u k}{2}} \omega^{\frac{u}{2}}-\omega^{\frac{-u k}{2}} \omega^{\frac{-u}{2}}\right) \omega^{\frac{-u}{2}}\left(\omega^{-u\left(\frac{n+j}{2}\right)}\right)\left(1+\omega^{u}\right)^{n} \text { by factoring } \\
& =\frac{1}{H+1} \sum_{u=1}^{H+1}\left(\omega^{u\left(\frac{k+1}{2}\right)}-\omega^{-u\left(\frac{k+1}{2}\right)}\right)\left(\omega^{-u\left(\frac{j+1}{2}\right)}\right)\left(\omega^{\frac{-u}{2}}\right)^{n}\left(1+\omega^{u}\right)^{n} \\
& =\frac{1}{H+1} \sum_{u=1}^{H+1}(2 i) \sin \left(\frac{u \pi(k+1)}{H+1}\right)\left(\cos \left(\frac{u \pi(j+1)}{H+1}\right)-i \sin \left(\frac{u \pi(j+1)}{H+1}\right)\right)\left(2 \cos \left(\frac{u \pi}{H+1}\right)\right)^{n}
\end{aligned}
$$

by substituting $\omega=\exp \left\{\frac{2 \pi i}{H+1}\right\}$ and using Euler's relation. The real part of this last expression equals $\left|L_{j, k}^{(n)}(H)\right|$ itself a real number, therefore

$$
\left|L_{j, k}^{(n)}(H)\right|=\frac{2}{H+1} \sum_{u=1}^{H+1} \sin \left(\frac{u \pi(k+1)}{H+1}\right) \sin \left(\frac{u \pi(j+1)}{H+1}\right)\left(2 \cos \left(\frac{u \pi}{H+1}\right)\right)^{n}
$$

For reference, we state this result as the following proposition. According to Mohanty (1979), a form of this result also appears in Kemperman (1961).

Proposition 1. Assume $j$ and $k$ are states in the following set $\{0,1,2, \ldots, H-1\}$ and suppose $L_{j, k}^{(n)}(H)$ is the collection of all sample paths going from state $j$ to state $k$ in $n$-steps in Figure 14. These sample paths correspond to lattice paths that move up or down by one unit each step and are confined to the horizontal strip bounded by lines $y=-1$ and $y=H$, never touching either of these boundaries, see Figure 15. Then the number of lattice paths in $L_{j, k}^{(n)}(H)$ denoted by $\left|L_{j, k}^{(n)}(H)\right|$ is given by the following expression.

$$
\left|L_{j, k}^{(n)}(H)\right|=\frac{2}{H+1} \sum_{u=1}^{H+1} \sin \left(\frac{u \pi(k+1)}{H+1}\right) \sin \left(\frac{u \pi(j+1)}{H+1}\right)\left(2 \cos \left(\frac{u \pi}{H+1}\right)\right)^{n} .
$$

We now use our path counting result to determine the n-step transition probability of the randomized Markov chain pictured in Figure 14, see Lemma 2. Recall this Markov chain originates from the dual process. Transient probability functions for the transient states of the dual process will then follow as a consequence of randomization in Lemma 3. A special case 


\section{Dual Processes To Solve Single Server Systems}

concerning the transient probability function of the dual process having absorbing state at -1 is then presented in Lemma 4.

Lemma 2. Suppose $0 \leq j \leq H-1$ and $0 \leq k \leq H-1$. The n-step transition probabilities, $P_{j, k}^{*(n)}$, for the Markov chain corresponding to Figure14 is given by the following expression.

$$
P_{j, k}^{*(n)}=\frac{2}{H+1} \sum_{u=1}^{H+1} \sin \left(\frac{u \pi(k+1)}{H+1}\right) \sin \left(\frac{u \pi(j+1)}{H+1}\right)\left(2 \cos \left(\frac{u \pi}{H+1}\right)\right)^{n} q^{\frac{n+k-j}{2}} p^{\frac{n-k+j}{2}}
$$

where $p=\frac{\lambda}{\lambda+\mu}$ and $q=\frac{\mu}{\lambda+\mu}$.

Proof. By the analysis following Figure 15,

$$
P_{j, k}^{*(n)}=\left|L_{j, k}^{(n)}(H)\right| q^{u} p^{d}
$$

where $u$ and $d$ are the number of upward and downward steps in getting from state $j$ to state $k$ in $n$ steps. The formula from Proposition 1 for $\left|L_{j, k}^{(n)}(H)\right|$ gives the result.

Lemma 3. Suppose $0 \leq j \leq H-1$ and $0 \leq k \leq H-1$, then the transition probability functions, $P_{j, k}^{*}(t)$, for the dual Markov process corresponding to Figure 13 is given by the following expression.

$$
\begin{aligned}
& P_{j, k}^{*}(t)=\frac{2}{H+1} q^{\frac{k-j}{2}} p^{\frac{-k+j}{2}} \underset{u=1}{H+1} \sin \left(\frac{u \pi(k+1)}{H+1}\right) \sin \left(\frac{u \pi(j+1)}{H+1}\right)\left[\exp \left\{(\lambda+\mu)\left(2 \sqrt{p q} \cos \left(\frac{u \pi}{H+1}\right)-1\right) t\right\}\right] \\
& =\frac{2}{H+1} q^{\frac{k-j}{2}} p^{\frac{-k+j}{2}} \sum_{u=1}^{H+1} \sin \left(\frac{u \pi(k+1)}{H+1}\right) \sin \left(\frac{u \pi(j+1)}{H+1}\right)\left[\exp \left\{\left(2 \sqrt{\lambda \mu} \cos \left(\frac{u \pi}{H+1}\right)-(\lambda+\mu)\right) t\right\}\right]
\end{aligned}
$$

where $p=\frac{\lambda}{\lambda+\mu}$ and $q=\frac{\mu}{\lambda+\mu}$.

Proof. By Theorem 2 (Randomization) and Lemma 2,

$$
P_{j, k}^{*}(t)=e^{-(\lambda+\mu) t} \sum_{n=0}^{\infty} \frac{[(\lambda+\mu) t]^{n}}{n !}\left[\frac{2}{H+1} \sum_{u=1}^{H+1} \sin \left(\frac{u \pi(k+1)}{H+1}\right) \sin \left(\frac{u \pi(j+1)}{H+1}\right)\left(2 \cos \left(\frac{u \pi}{H+1}\right)\right)^{n}\right] q \frac{n+k-j}{2} q^{\frac{n-k+j}{2}}
$$




$$
\begin{aligned}
& =e^{-(\lambda+\mu) t} \frac{2}{H+1} q^{\frac{k-j}{2}} p^{\frac{-k+j}{2}}\left[\sum_{u=1}^{H+1} \sin \left(\frac{u \pi(k+1)}{H+1}\right) \sin \left(\frac{u \pi(j+1)}{H+1}\right) \sum_{n=0}^{\infty} \frac{[(\lambda+\mu) t]^{n}}{n !}\left(2 \cos \left(\frac{u \pi}{H+1}\right)\right)^{n}(\sqrt{p q})^{n}\right] \\
& =\frac{2}{H+1} q^{\frac{k-j}{2}} \frac{-k+j}{2}\left[\sum_{u=1}^{H+1} \sin \left(\frac{u \pi(k+1)}{H+1}\right) \sin \left(\frac{u \pi(j+1)}{H+1}\right)\left[\exp \left\{(\lambda+\mu)\left(2 \sqrt{p q} \cos \left(\frac{u \pi}{H+1}\right)-1\right) t\right\}\right]\right]
\end{aligned}
$$

by the Taylor series for $\exp \{x\}=\sum_{n=0}^{\infty} \frac{x^{n}}{n !}$.

Lemma 4. Suppose $0 \leq j \leq H-1$, then the transition probability functions, $P_{j,-1}^{*}(t)$, for the dual Markov process corresponding to Figure 13 is given by the following expression.

$$
P_{j,-1}^{*}(t)=\frac{2 \lambda}{H+1} q^{\frac{-j}{2}} \underset{p^{2}}{\stackrel{j}{2} \underset{u}{H}+1}\left[\left(2 \sqrt{\lambda \mu} \cos \left(\frac{u \pi}{H+1}\right)-(\lambda+\mu)\right)\right]^{-1} \sin \left(\frac{u \pi(j+1)}{H+1}\right) \sin \left(\frac{u \pi}{H+1}\right)\left[\exp \left\{\left(2 \sqrt{\lambda \mu} \cos \left(\frac{u \pi}{H+1}\right)-(\lambda+\mu)\right) t\right\}-1\right]
$$

Proof. Kolmogorov's forward equation, $\frac{d}{d t} P^{*}(t)=P^{*}(t) \cdot Q^{*}$ implies that $\frac{d}{d t} P_{j,-1}^{*}(t)=\lambda P_{j, 0}^{*}(t)$. Lemma 4 follows by substituting into this equation the expression given for $P_{j, 0}^{*}(t)$ from Lemma 3 and integrating term by term with respect to t. The constant of integration is determined by the initial value $P_{j,-1}^{*}(0)=0$ when $j>-1$.

Having determined the transient probability functions of the dual process, we are now in position to find the transient probability functions of the original $\mathrm{M} / \mathrm{M} / 1 / \mathrm{H}$ queueing system.

Theorem 3. The transient probability functions $P_{i, j}(t)$ of the $\mathrm{M} / \mathrm{M} / 1 / \mathrm{H}$ queueing system corresponding to Figure 11 are as follows.

Case $1 . \quad 1 \leq i \leq H$ and $1 \leq j \leq H-1$

$$
\begin{aligned}
& P_{i, j}(t)= \\
& \frac{2 \lambda}{H+1} \underset{u=1}{H+1}\left[q \frac{-j+1}{2} \frac{j-1}{2} \sin \left(\frac{u \pi(j)}{H+1}\right)-q^{2} \frac{-j}{p^{2}} \sin \left(\frac{u \pi(j+1)}{H+1}\right)\right] \sin \left(\frac{u \pi}{H+1}\right)\left(2 \sqrt{\lambda \mu} \cos \left(\frac{u \pi}{H+1}\right)-(\lambda+\mu)\right)^{-1}\left[\exp \left\{\left(2 \sqrt{\lambda \mu} \cos \left(\frac{u \pi}{H+1}\right)-(\lambda+\mu)\right) t\right\}-1\right] \\
& +\frac{2}{H+1} \underset{u=1}{H+1} \sum_{k=0}^{i-1}\left(\frac{k-j+1}{2} \frac{-k+j-1}{2} \sin \left(\frac{u \pi(j)}{H+1}\right)-q^{\frac{k-j}{2}} \frac{-k+j}{2} \sin \left(\frac{u \pi(j+1)}{H+1}\right)\right) \sin \left(\frac{u \pi(k+1)}{H+1}\right) \exp \left\{\left(2 \sqrt{\lambda \mu} \cos \left(\frac{u \pi}{H+1}\right)-(\lambda+\mu)\right) t\right\}
\end{aligned}
$$


Dual Processes To Solve Single Server Systems

Case 2. $\quad i=0$ and $1 \leq j \leq H-1$

$$
\begin{aligned}
& P_{0, j}(t)= \\
& \frac{2 \lambda}{H+1} \underset{u=1}{H+1}\left[\frac{-j+1}{2} \frac{j-1}{2} \sin \left(\frac{u \pi(j)}{H+1}\right)-q^{\frac{-j}{2}} \frac{j}{p^{2}} \sin \left(\frac{u \pi(j+1)}{H+1}\right)\right] \sin \left(\frac{u \pi}{H+1}\right)\left(2 \sqrt{\lambda \mu} \cos \left(\frac{u \pi}{H+1}\right)-(\lambda+\mu)\right)^{-1}\left[\exp \left\{\left(2 \sqrt{\lambda \mu} \cos \left(\frac{u \pi}{H+1}\right)-(\lambda+\mu)\right) t\right\}-1\right]
\end{aligned}
$$

Case 3. $\quad 1 \leq i \leq H$ and $j=0$

$$
\begin{aligned}
& P_{i, 0}(t)=1-\frac{2 \lambda}{H+1} \sum_{u=1}^{H+1} \sin ^{2}\left(\frac{u \pi}{H+1}\right)\left(2 \sqrt{\lambda \mu} \cos \left(\frac{u \pi}{H+1}\right)-(\lambda+\mu)\right)^{-1}\left[\exp \left\{\left(2 \sqrt{\lambda \mu} \cos \left(\frac{u \pi}{H+1}\right)-(\lambda+\mu)\right) t\right\}-1\right]- \\
& \frac{2}{H+1} \sum_{u=1}^{H+1} \sum_{k=0}^{i-1}\left(q^{2} p^{\frac{k}{2}} \sin \left(\frac{u \pi(k+1)}{H+1}\right)\right) \sin \left(\frac{u \pi}{H+1}\right) \exp \left\{\left(2 \sqrt{\lambda \mu} \cos \left(\frac{u \pi}{H+1}\right)-(\lambda+\mu)\right) t\right\}
\end{aligned}
$$

Case 4. $\quad i=0$ and $j=0$

$$
P_{0,0}(t)=1-\frac{2 \lambda}{H+1} \sum_{u=1}^{H+1} \sin ^{2}\left(\frac{u \pi}{H+1}\right)\left(2 \sqrt{\lambda \mu} \cos \left(\frac{u \pi}{H+1}\right)-(\lambda+\mu)\right)^{-1}\left[\exp \left\{\left(2 \sqrt{\lambda \mu} \cos \left(\frac{u \pi}{H+1}\right)-(\lambda+\mu)\right)\right\}-1\right]
$$

Case 5. $\quad 1 \leq i \leq H$ and $j=H$

$$
\begin{aligned}
& P_{i, H}(t)= \\
& \frac{2 \lambda}{H+1} \underset{u=1}{H+1}\left[\frac{-H+1}{2} \frac{H-1}{2} \sin \left(\frac{u \pi H}{H+1}\right)\right] \sin \left(\frac{u \pi}{H+1}\right)\left(2 \sqrt{\lambda \mu} \cos \left(\frac{u \pi}{H+1}\right)-(\lambda+\mu)\right)^{-1}\left[\exp \left\{\left(2 \sqrt{\lambda \mu} \cos \left(\frac{u \pi}{H+1}\right)-(\lambda+\mu)\right)\right\}-1\right]+ \\
& \frac{2}{H+1} \underset{u=1}{H+1} \sum_{k=0}^{i-1}(\frac{k-H+1}{2} \underbrace{\frac{-k+H-1}{2}} \sin \left(\frac{u \pi(k+1)}{H+1}\right)) \sin \left(\frac{u \pi H}{H+1}\right) \exp \left\{\left(2 \sqrt{\lambda \mu} \cos \left(\frac{u \pi}{H+1}\right)-(\lambda+\mu)\right)\right\}
\end{aligned}
$$

Case 6. $\quad i=0$ and $j=H$

$$
P_{0, H}(t)=\frac{2 \lambda}{H+1} \underset{u=1}{H+1}{ }^{H} \frac{-H+1}{2} \frac{p^{2}}{2-1} \sin \left(\frac{u \pi H}{H+1}\right) \sin \left(\frac{u \pi}{H+1}\right)\left(2 \sqrt{\lambda \mu} \cos \left(\frac{u \pi}{H+1}\right)-(\lambda+\mu)\right)^{-1}\left[\exp \left\{\left(2 \sqrt{\lambda \mu} \cos \left(\frac{u \pi}{H+1}\right)-(\lambda+\mu)\right)\right\}-1\right]
$$




\section{Proof.}

\section{Cases 1 and 2.}

The result follows from $P_{i, j}(t)=\left[P_{j-1,-1}^{*}(t)-P_{j,-1}^{*}(t)\right]+\sum_{k=0}^{i-1}\left[P_{j-1, k}^{*}(t)-P_{j, k}^{*}(t)\right]$ found in Corollary 1 and using Lemmas 3 and 4 . In Case 2, the summation term is missing.

Cases 3 and 4.

Corollary 1 becomes $P_{i, 0}(t)=1-P_{0,-1}^{*}(t)-\sum_{k=0}^{i-1}\left[P_{0, k}^{*}(t)\right]$ because -1 is an absorbing state. The formula then follows as before by substituting expressions from Lemmas 3 and 4 . In Case 4, the summation term is gone.

\section{Cases 5 and 6.}

Corollary 1 is now $P_{i, H}(t)=P_{H-1,-1}^{*}(t)+\sum_{k=0}^{i-1}\left[P_{H-1, k}^{*}(t)\right]$ because $H$ is an absorbing state and once again Lemmas 3 and 4 produce the preceding expression. In Case 6, the summation term is gone.

Theorem 4 (Takacs). The transient probability functions $P_{i, j}(t)$ of the $\mathrm{M} / \mathrm{M} / 1 / \mathrm{H}$ queueing system corresponding to Figure 11 are as follows.

If $\lambda \neq \mu$ then

$$
\begin{aligned}
& P_{i, j}(t)=\frac{1-\left(\frac{\lambda}{\mu}\right)}{\left.1-\left(\frac{\lambda}{\mu}\right)^{H+1}\left(\frac{\lambda}{\mu}\right)^{j}+\frac{2 \mu}{H+1}\left(\frac{\lambda}{\mu}\right)^{\frac{j-i}{2}} \sum_{u=1}^{H} \exp \left\{\left(2 \sqrt{\lambda \mu} \cos \left(\frac{u \pi}{H+1}\right)-(\lambda+\mu)\right)\right\}\right\}\left((\lambda+\mu)-2 \sqrt{\lambda \mu} \cos \left(\frac{u \pi}{H+1}\right)\right)^{-1} .} \\
& {\left[\sin \left(\frac{u \pi i}{H+1}\right)-\sqrt{\frac{\lambda}{\mu}} \sin \left(\frac{u \pi(i+1)}{H+1}\right)\right]\left[\sin \left(\frac{u \pi j}{H+1}\right)-\sqrt{\frac{\lambda}{\mu}} \sin \left(\frac{u \pi(j+1)}{H+1}\right)\right] }
\end{aligned}
$$

If $\lambda=\mu$ then

$$
\left.P_{i, j}(t)=\frac{1}{H+1}+\frac{1}{H+1} \sum_{u=1}^{H} \exp \left\{\left(2 \lambda \cos \left(\frac{u \pi}{H+1}\right)-2 \lambda\right)\right\}\right\}\left(1-\cos \left(\frac{u \pi}{H+1}\right)\right)^{-1}\left[\sin \left(\frac{u \pi i}{H+1}\right)-\sin \left(\frac{u \pi(i+1)}{H+1}\right)\right]\left[\sin \left(\frac{u \pi j}{H+1}\right)-\sin \left(\frac{u \pi(j+1)}{H+1}\right)\right]
$$

Proof. The constant term in this theorem is most easily obtained by realizing it corresponds to the well-known steady state solution of the $\mathrm{M} / \mathrm{M} / 1 / \mathrm{H}$ queueing system, see Gross and Harris (1985). This constant term is also associated with the 0 eigenvalue of this system, see Krinik and Mortensen (2004). The remaining portion of the transient probability function may be derived through algebraic manipulations of the solutions found in Theorem 3. In particular, the following geometric series identity, 


$$
\sum_{k=1}^{i} a^{k} \sin (\varpi k)=\frac{a \sin (\varpi)-a^{i+1} \sin (\varpi(i+1))+a^{i+2} \sin (\varpi i)}{a^{2}-2 a \cos (\varpi)+1}
$$

is useful in reducing the double sums appearing in Theorem 3 to a single sum expression. The remaining details may be found in Kasfy (2004).

It is interesting to compare Theorem 3 and its derivation to the traditional method of solution see, for example, Theorem 1 on pages 13-21 of Takacs (1962) where $P_{i, j}(t)$ is determined using a linear algebraic, eigenvalue approach. In contrast, our approach here uses dual processes, randomization, lattice path combinatorics (Proposition 1) and the Taylor series expansion of the exponential function.

\section{5. $\mathrm{M} / \mathrm{M} / \mathbf{1} / \mathbf{H}$ with Catastrophes}

In this section the classical $\mathrm{M} / \mathrm{M} / 1 / \mathrm{H}$ queueing system is modified to include a constant catastrophe rate, $\gamma$, which goes to state 0 . Catastrophes are assumed possible at any non-zero state, but with a constant rate, and when they occur, they reduce the queueing system to zero customers. The transitions are shown in the following state rate transition diagram, Figure 16.

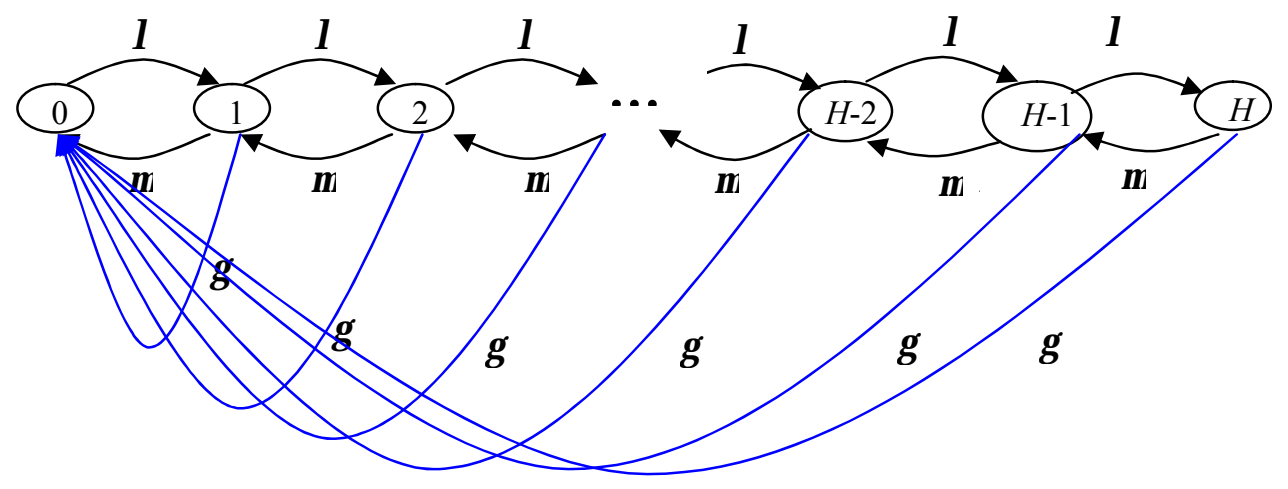

Figure 16

Although the usual transition matrix of this system has dimension $H+1$ by $H+1$, we represent transitions in an augmented $H+2$ by $H+2$ matrix having rates as shown below. The reason for such an enlarged Q-matrix is in anticipation of finding its dual process. 
Dual Processes To Solve Single Server Systems

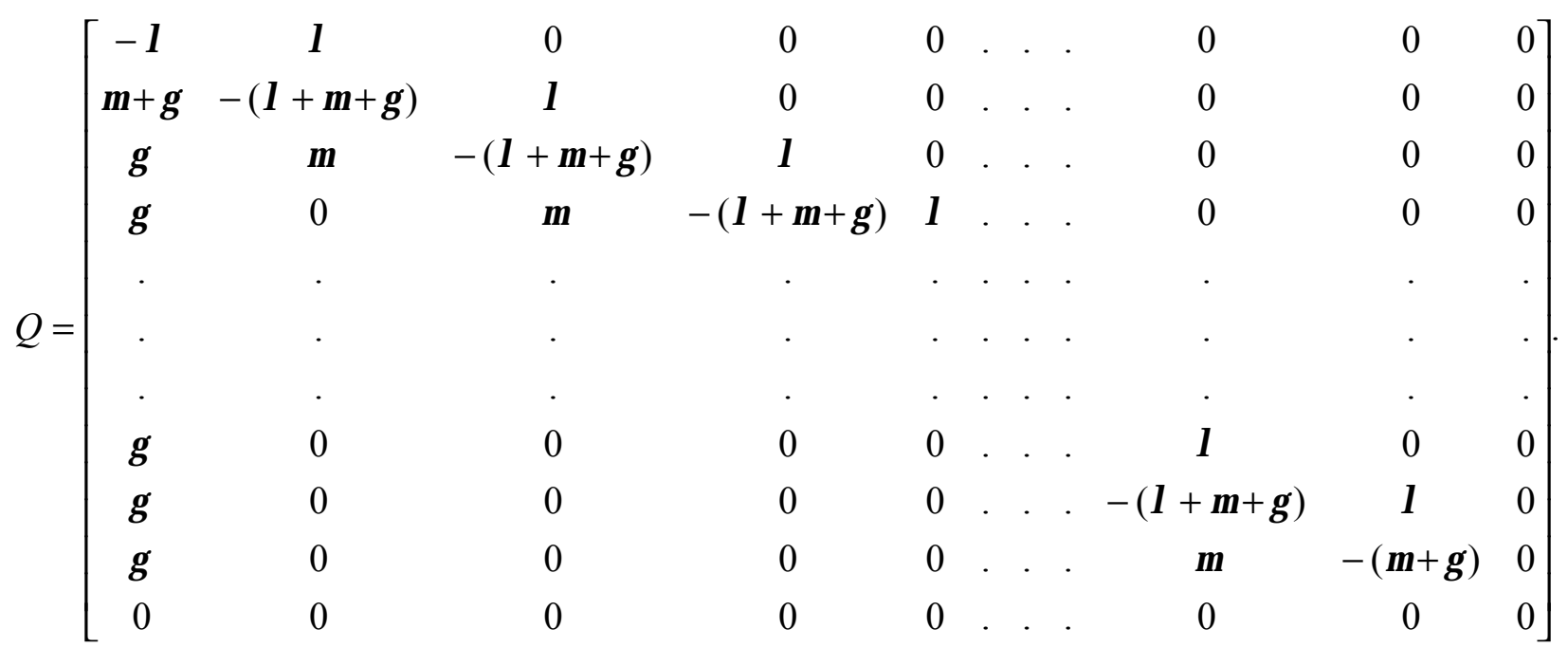

As before, the objective is to determine the unique transient probability functions, $P_{i, j}(t)$, corresponding to Q. Fortunately, the theory of dual processes has been developed past birthdeath processes and there is an extension of Corollary 1. Specifically, by a result of Kirstein (see Anderson (1991), Theorem 3.4, pages 248-249), $P_{i, j}(t)$ is stochastically monotone for the $\mathrm{M} / \mathrm{M} / 1 / \mathrm{H}$ with catastrophe process and therefore by a theorem of Siegmund (see Anderson (1991), the argument within the proof of Proposition 4.1 and the remarks following the proof on pages 251-252), it follows that

$$
P_{i, j}(t)=\sum_{k=0}^{i}\left[P_{j, k}^{*}(t)-P_{j+1, k}^{*}(t)\right]
$$

where $P_{j, k}^{*}(t)$ is the unique transition probability function corresponding to the dual process having infinitesimals rates $Q^{*}$ given by

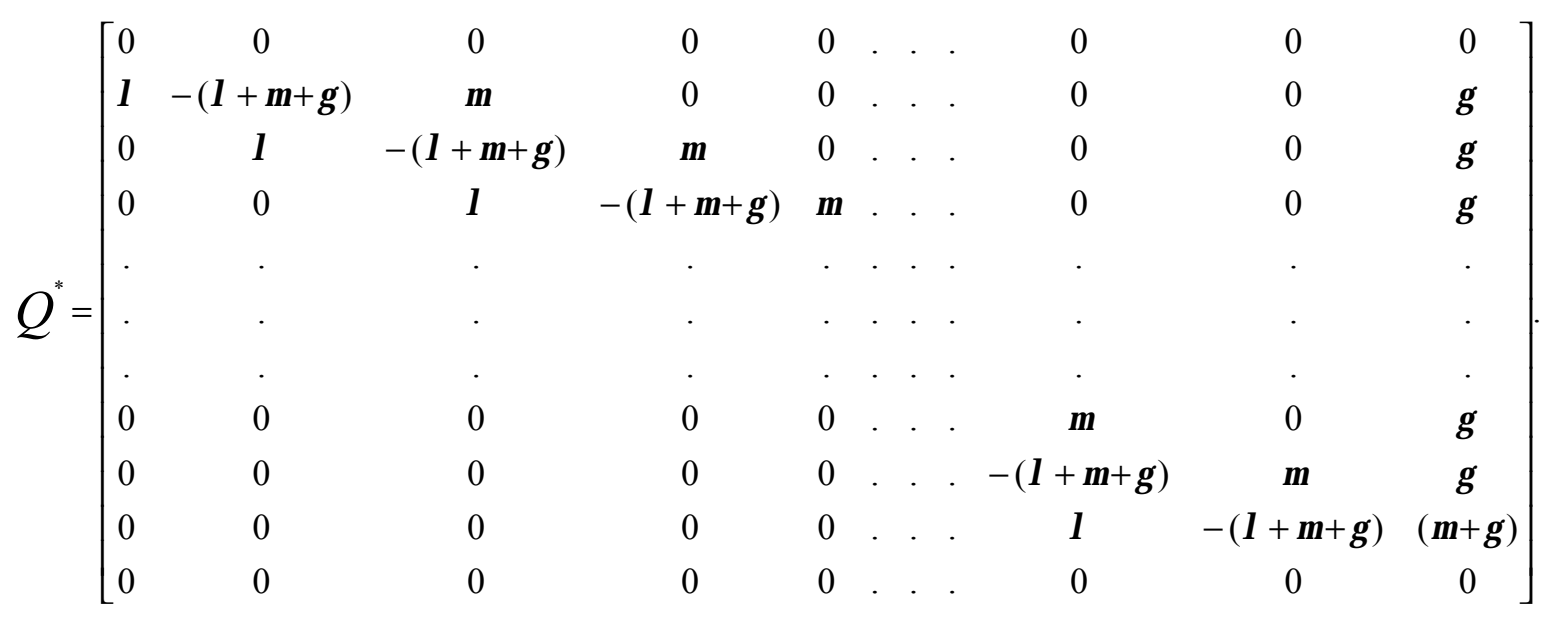


In this section, we will refer to $P_{i, j}(t)=\sum_{k=0}^{i}\left[P_{j, k}^{*}(t)-P_{j+1, k}^{*}(t)\right]$ as Siegmund's equation. The state space of the dual process is $\{0,1,2, \ldots, H+1\}$ where " $H+1$ " is the new added state (instead of "-1" as in section 2). In this way, Siegmund's equation, which connects the transition probability functions of the original and dual processes, is seen to be the same as Corollary 1 shifted to the right by a unit. The dual process has transition rate diagram as given in Figure 17.

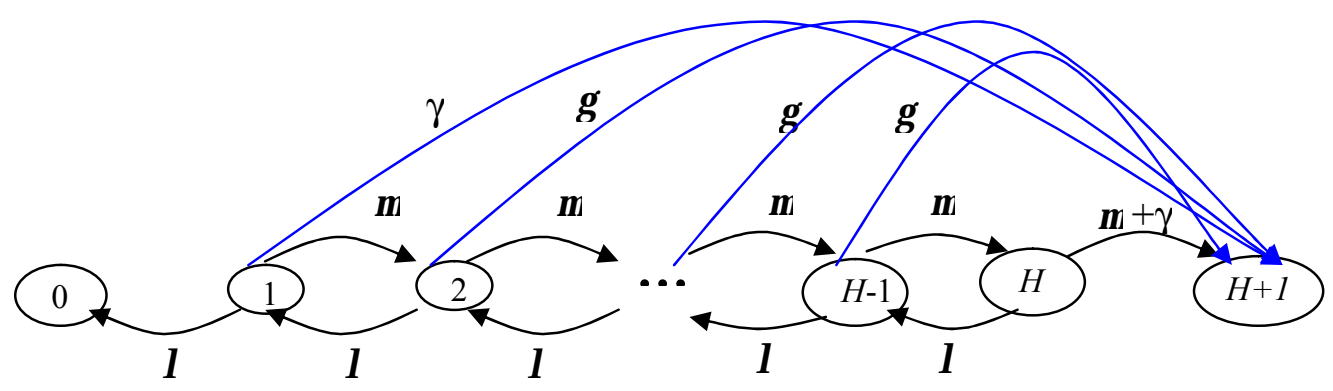

Figure 17

Randomizing this system gives the following randomized Markov Chain of the dual process.

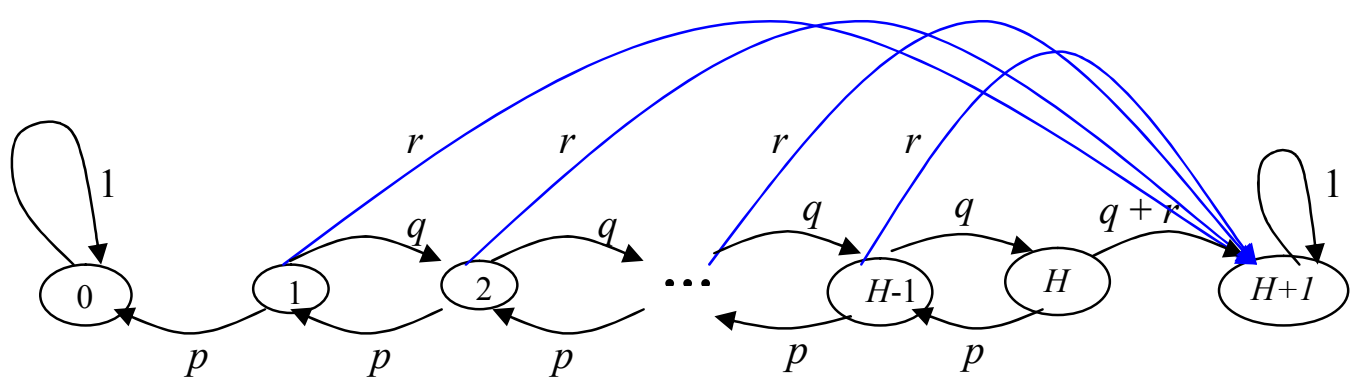

Figure 18

where

$$
p=\frac{\lambda}{\gamma+\lambda+\mu}, \quad q=\frac{\mu}{\gamma+\lambda+\mu}, \quad r=\frac{\gamma}{\gamma+\lambda+\mu} .
$$

Noticing the similarity between Figures 14 and 18, the following results in analogy with the theorems of section 4 may be deduced. 


\section{Dual Processes To Solve Single Server Systems}

Lemma 5. Suppose $1 \leq j \leq H$ and $1 \leq k \leq H$. The n-step transition probabilities, $P_{j, k}^{*(n)}$, for the Markov chain corresponding to Figure 18 is given by the following expression.

$$
P_{j, k}^{*(n)}=\frac{2}{H+1} \underset{u=1}{H+1} \sin \left(\frac{u \pi k}{H+1}\right) \sin \left(\frac{u \pi j}{H+1}\right)\left(2 \cos \left(\frac{u \pi}{H+1}\right)\right)^{n} q^{\frac{n+k-j}{2}} p^{\frac{n-k+j}{2}}
$$

with $p=\frac{\lambda}{\gamma+\lambda+\mu}, q=\frac{\mu}{\gamma+\lambda+\mu}$.

Proof. The number of lattice paths from state $j$ to state $k$ restricted to the horizontal band strictly between 0 and $H+1$ equals the number of lattice paths from state $(j-1)$ to state $(k-1)$ confined to a horizontal band strictly between -1 and $H$. However by Proposition 1 , there are $\frac{2}{H+1} \sum_{u=1}^{H+1} \sin \left(\frac{u \pi k}{H+1}\right) \sin \left(\frac{u \pi j}{H+1}\right)\left(2 \cos \left(\frac{u \pi}{H+1}\right)\right)^{n}$ such paths. The proof now proceeds as in Lemma 2.

Lemma 6. Suppose $1 \leq j \leq H$ and $1 \leq k \leq H$, then the transition probability functions, $P_{j, k}^{*}(t)$ for the dual Markov process corresponding to Figure 17 is given by the following expression.

$$
P_{j, k}^{*}(t)=\frac{2}{H+1} q^{\frac{k-j}{2}} p^{\frac{-k+j}{2}} \sum_{u=1}^{H+1} \sin \left(\frac{u \pi k}{H+1}\right) \sin \left(\frac{u \pi j}{H+1}\right) \exp \left\{\left(2 \sqrt{\lambda \mu} \cos \left(\frac{u \pi}{H+1}\right)-(\lambda+\mu+\gamma)\right) t\right\}
$$

where $p=\frac{\lambda}{\gamma+\lambda+\mu}, q=\frac{\mu}{\gamma+\lambda+\mu}$

Proof. By Theorem 2 (Randomization) and Lemma 5,

$$
\begin{aligned}
& P_{j, k}^{*}(t)=e^{-(\lambda+\mu+\gamma) t} \sum_{n=0}^{\infty} \frac{[(\lambda+\mu+\gamma) t]^{n}}{n !}\left[\frac{2}{H+1} \sum_{u=1}^{H+1} \sin \left(\frac{u \pi k}{H+1}\right) \sin \left(\frac{u \pi j}{H+1}\right)\left(2 \cos \left(\frac{u \pi}{H+1}\right)\right)^{n}\right] q^{\frac{n+k-j}{2}} p^{\frac{n-k+j}{2}} \\
& =e^{-(\lambda+\mu+\gamma) t} \frac{2}{H+1} q^{\frac{k-j}{2}} \frac{-k+j}{2}\left[\underset{\sum=1}{H+1} \sin \left(\frac{u \pi k}{H+1}\right) \sin \left(\frac{u \pi j}{H+1}\right) \sum_{n=0}^{\infty} \frac{[(\lambda+\mu+\gamma) t]^{n}}{n !}\left(2 \cos \left(\frac{u \pi}{H+1}\right)\right)^{n}(\sqrt{p q})^{n}\right]
\end{aligned}
$$

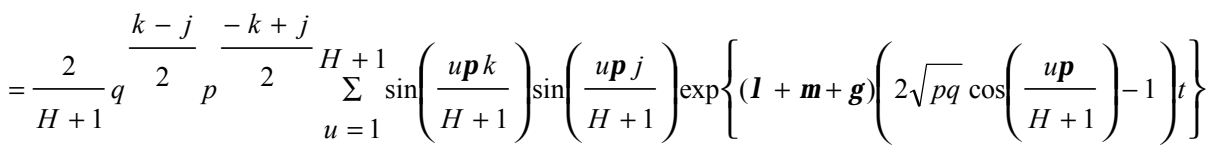


by the Taylor series for $\exp \{x\}=\sum_{n=0}^{\infty} \frac{x^{n}}{n !}$.

Lemma 7. Suppose $1 \leq j \leq H$, then the transition probability functions, $P_{j, 0}^{*}(t)$, for the dual Markov process corresponding to Figure 17 is given by the following expression.

$$
\begin{aligned}
& P_{j, 0}^{*}(t)= \\
& \frac{2 \lambda}{H+1} q{ }^{\frac{1-j}{2}} \frac{-1+j}{2} \underset{\sum_{u=1}}{H+1}\left[\left(2 \sqrt{\lambda \mu} \cos \left(\frac{u \pi}{H+1}\right)-(\lambda+\mu+\gamma)\right]^{-1} \sin \left(\frac{u \pi j}{H+1}\right) \sin \left(\frac{u \pi}{H+1}\right)\left[\exp \left\{\left(2 \sqrt{\lambda \mu} \cos \left(\frac{u \pi}{H+1}\right)-(\lambda+\mu+\gamma)\right) t\right\}-1\right]\right.
\end{aligned}
$$

Proof. Kolmogorov's forward equation, $\frac{d}{d t} P^{*}(t)=P^{*}(t) \cdot Q^{*}$ implies that $\frac{d}{d t} P_{j, 0}^{*}(t)=\lambda P_{j, 1}^{*}(t)$. $P_{j, 1}^{*}(t)$ is known from Lemma 6 and $P_{j, 0}^{*}(t)$ may be determined by integrating with respect to $t$ as in Lemma 4. The constant of integration is once again determined by the initial value $P_{j, 0}^{*}(t)=0$ which holds for $j>0$.

Again having determined the transient probability functions of the dual process, we may now substitute into Siegmund's equation, $P_{i, j}(t)=\sum_{k=0}^{i}\left[P_{j, k}^{*}(t)-P_{j+1, k}^{*}(t)\right]$, to find the transient probability functions of the $\mathrm{M} / \mathrm{M} / 1 / \mathrm{H}$ with catastrophe queueing system.

Theorem 5. The transient probability functions, $P_{i, j}(t)$, of the $\mathrm{M} / \mathrm{M} / 1 / \mathrm{H}$ system with catastrophes corresponding to Figure 16 are as follows, where $p=\frac{\lambda}{\gamma+\lambda+\mu}, q=\frac{\mu}{\gamma+\lambda+\mu}$.

Case $1 . \quad 1 \leq i \leq H$ and $1 \leq j \leq H-1$

$$
\begin{aligned}
& P_{i, j}(t)= \\
& \frac{2 \lambda}{H+1} \sum_{u=1}^{H+1}\left[\frac{1-j}{2} \frac{-1+j}{2} \sin \left(\frac{u \pi j}{H+1}\right)-q^{\frac{-j}{2}} \frac{j}{p^{2}} \sin \left(\frac{u \pi(j+1)}{H+1}\right)\right] \sin \left(\frac{u \pi}{H+1}\right)\left(2 \sqrt{\lambda \mu} \cos \left(\frac{u \pi}{H+1}\right)-(\lambda+\mu+\gamma)\right)^{-1} . \\
& {\left[\exp \left\{\left(2 \sqrt{\lambda \mu} \cos \left(\frac{u \pi}{H+1}\right)-(\lambda+\mu+\gamma)\right) t\right\}-1\right]+\frac{2}{H+1} \sum_{u=1}^{H+1} \sum_{k=1}^{i}\left(\frac{k-j}{2} \frac{-k+j}{2} \sin \left(\frac{u \pi j}{H+1}\right)-q \frac{k-j-1}{2} \frac{-k+j+1}{2} \sin \left(\frac{u \pi(j+1)}{H+1}\right)\right) .} \\
& \sin \left(\frac{u \pi k}{H+1}\right) \exp \left\{\left(2 \sqrt{\lambda \mu} \cos \left(\frac{u \pi}{H+1}\right)-(\lambda+\mu+\gamma)\right) t\right\}
\end{aligned}
$$


Case 2. $\quad i=0$ and $1 \leq j \leq H-1$

$$
\begin{aligned}
P_{0, j}(t)= & \frac{2 \lambda}{H+1} \sum_{u=1}^{H+1}\left[\frac{1-j}{2} \frac{-1+j}{2} \sin \left(\frac{u \pi j}{H+1}\right)-q^{\frac{-j}{2}} \frac{j}{p^{2}} \sin \left(\frac{u \pi(j+1)}{H+1}\right)\right] \sin \left(\frac{u \pi}{H+1}\right) \\
& \left(2 \sqrt{\lambda \mu} \cos \left(\frac{u \pi}{H+1}\right)-(\lambda+\mu+\gamma)\right)^{-1}\left[\exp \left\{\left(2 \sqrt{\lambda \mu} \cos \left(\frac{u \pi}{H+1}\right)-(\lambda+\mu+\gamma)\right) t\right\}-1\right]
\end{aligned}
$$

Case 3. $\quad 1 \leq i \leq H$ and $j=0$

$$
\begin{aligned}
& P_{i, 0}(t)= \\
& 1-\frac{2 \lambda}{H+1} \sum_{u=1}^{H+1} \sin ^{2}\left(\frac{u \pi}{H+1}\right)\left(2 \sqrt{\lambda \mu} \cos \left(\frac{u \pi}{H+1}\right)-(\lambda+\mu+\gamma)\right)^{-1} \cdot\left[\exp \left\{\left(2 \sqrt{\lambda \mu} \cos \left(\frac{u \pi}{H+1}\right)-(\lambda+\mu+\gamma)\right) t\right\}-1\right] \\
& -\frac{2}{H+1} \sum_{k=1}^{i} q^{\frac{k-1}{2}} p^{\frac{-k+1}{2}} \sum_{u=1}^{H+1} \sin \left(\frac{u \pi k}{H+1}\right) \sin \left(\frac{u \pi}{H+1}\right) \exp \left\{\left(2 \sqrt{\lambda \mu} \cos \left(\frac{u \pi}{H+1}\right)-(\lambda+\mu+\gamma)\right) t\right\}
\end{aligned}
$$

Case 4. $\quad i=0$ and $j=0$

$$
P_{0,0}(t)=1-\frac{2 \lambda}{H+1} \sum_{u=1}^{H+1} \sin ^{2}\left(\frac{u \pi}{H+1}\right)\left(2 \sqrt{\lambda \mu} \cos \left(\frac{u \pi}{H+1}\right)-(\lambda+\mu+\gamma)\right)^{-1} \cdot\left[\exp \left\{\left(2 \sqrt{\lambda \mu} \cos \left(\frac{u \pi}{H+1}\right)-(\lambda+\mu+\gamma)\right) t\right\}-1\right]
$$

Case 5. $\quad 1 \leq i \leq H$ and $j=H$

$$
\begin{aligned}
& P_{i, H}(t)= \\
& \frac{2 \lambda}{H+1} \sum_{u=1}^{H+1}\left[q \frac{1-H}{2} \frac{-1+H}{2} \sin \left(\frac{u \pi H}{H+1}\right)\right] \sin \left(\frac{u \pi}{H+1}\right)\left(2 \sqrt{\lambda \mu} \cos \left(\frac{u \pi}{H+1}\right)-(\lambda+\mu)\right)^{-1}\left[\exp \left\{\left(2 \sqrt{\lambda \mu} \cos \left(\frac{u \pi}{H+1}\right)-(\lambda+\mu)\right)\right\}-1\right]+ \\
& \frac{2}{H+1} \sum_{u=1}^{H+1} \sum_{k=1}^{i} \frac{k-H}{2} \frac{-k+H}{2} \sin \left(\frac{u \pi k}{H+1}\right) \sin \left(\frac{u \pi H}{H+1}\right) \exp \left\{\left(2 \sqrt{\lambda \mu} \cos \left(\frac{u \pi}{H+1}\right)-(\lambda+\mu)\right) t\right\}
\end{aligned}
$$

Case 6. $\quad i=0$ and $j=H$

$$
\begin{aligned}
& P_{0, H}(t)= \\
& \frac{2 \lambda}{H+1} \underset{u=1}{H+1}\left[\frac{1-H}{2} \frac{-1+H}{2} \sin \left(\frac{u \pi H}{H+1}\right)\right] \sin \left(\frac{u \pi}{H+1}\right)\left(2 \sqrt{\lambda \mu} \cos \left(\frac{u \pi}{H+1}\right)-(\lambda+\mu)\right)^{-1}\left[\exp \left\{\left(2 \sqrt{\lambda \mu} \cos \left(\frac{u \pi}{H+1}\right)-(\lambda+\mu)\right)\right\}-1\right]
\end{aligned}
$$




\section{Dual Processes To Solve Single Server Systems}

\section{Proof.}

Cases 1 and 2.

The result follows from

$$
P_{i, j}(t)=\left[P_{j, 0}^{*}(t)-P_{j+1,0}^{*}(t)\right]+\sum_{k=1}^{i}\left[P_{j, k}^{*}(t)-P_{j+1, k}^{*}(t)\right]
$$

and using Lemmas 6 and 7. In Case 2, the summation term is gone.

Cases 3 and 4.

Siegmund's equation becomes $P_{i, 0}(t)=\left[1-P_{1,0}^{*}(t)\right]-\sum_{k=1}^{i} P_{1, k}^{*}(t)$ because 0 is an absorbing state. The formula then follows as before by substituting expressions from Lemmas 6 and 7. In Case 4, the summation term is missing.

\section{Cases 5 and 6.}

Now $P_{i, H}(t)=P_{H, 0}^{*}(t)+\sum_{k=1}^{i}\left[P_{H, k}^{*}(t)\right]$ because $H+1$ is an absorbing state and once again Lemmas 6 and 7 produce the preceding expression. In Case 6, the summation term is gone.

This completes the proof of Theorem 5 .

The close connection between the transient solutions of $\mathrm{M} / \mathrm{M} / 1 / \mathrm{H}$ and $\mathrm{M} / \mathrm{M} / 1 / \mathrm{H}$ with catastrophes becomes clear when looking at Figures 14 and 18. The path counting is the same, only the probabilities are re-normalized. Our path counting results would have appeared exactly the same for each system had Siegmund's equation been used throughout this article in place of Corollary 1. This leaves the natural remaining issue of whether Takac's Theorem 4 generalizes in a straightforward way to $\mathrm{M} / \mathrm{M} / 1 / \mathrm{H}$ with catastrophes? The answer is yes and is given in Theorem 6 which unifies the different cases of transient probability functions listed within Theorem 5.

Theorem 6. Suppose $\gamma, \lambda, \mu>0$ then the transient probability functions, $P_{i, j}(t)$, for $0 \leq i \leq H$ and $0 \leq j \leq H$, of the $\mathrm{M} / \mathrm{M} / 1 / \mathrm{H}$ with catastrophes system shown in Figure 16 are as follows.

$$
\begin{aligned}
& P_{i, j}(t)=A r_{1}^{j}+B r_{2}^{j}+\frac{2 \mu}{H+1}\left(\frac{\lambda}{\mu}\right)^{\frac{j-i}{2}} \sum_{u=1}^{H} \exp \left\{\left(2 \sqrt{\lambda \mu} \cos \left(\frac{u \pi}{H+1}\right)-(\gamma+\lambda+\mu)\right\}\right\}\left((\gamma+\lambda+\mu)-2 \sqrt{\lambda \mu} \cos \left(\frac{u \pi}{H+1}\right)\right)^{-1} . \\
& {\left[\sin \left(\frac{u \pi i}{H+1}\right)-\sqrt{\frac{\lambda}{\mu}} \sin \left(\frac{u \pi(i+1)}{H+1}\right)\right]\left[\sin \left(\frac{u \pi j}{H+1}\right)-\sqrt{\frac{\lambda}{\mu}} \sin \left(\frac{u \pi(j+1)}{H+1}\right)\right] }
\end{aligned}
$$




$$
\begin{aligned}
& \text { where } r_{1}=\frac{(\gamma+\lambda+\mu)+\sqrt{(\gamma+\lambda+\mu)^{2}-4 \lambda \mu}}{2 \mu} \text { and } r_{2}=\frac{(\gamma+\lambda+\mu)-\sqrt{(\gamma+\lambda+\mu)^{2}-4 \lambda \mu}}{2 \mu} \\
& A=\frac{\left(1-r_{1}\right)\left(1-r_{2}\right)\left(r_{2} \mu-\gamma-\lambda\right)+\gamma\left(1-r_{1}\right)\left(1-r_{2}^{H+1}\right)}{\left(1-r_{2}\right)\left(r_{2} \mu-\gamma-\lambda\right)\left(1-r_{1}^{H+1}\right)+\left(\gamma+\lambda-r_{1} \mu\right)\left(1-r_{1}\right)\left(1-r_{2}^{H+1}\right)} \text { and } B=\frac{\left(\gamma+\lambda-r_{1} \mu\right) A-\gamma}{r_{2} \mu-\gamma-\lambda}
\end{aligned}
$$

Proof. The $A r_{1}^{j}+B r_{2}^{j}$ term in this theorem corresponds to the steady state distribution of the $\mathrm{M} / \mathrm{M} / 1 / \mathrm{H}$ with catastrophes system. This distribution is determined by noting that the steady state distribution satisfies a linear, constant coefficient, second order recurrence relation. The remaining portion of the preceding transient probability function is derived through algebraic manipulations of the solutions found in Theorem 5 following the same arguments that produced Theorem 4 from Theorem 3; see Kasfy (2004) for complete details of this calculation.

\section{Remarks.}

1. It is intriguing to have two queueing systems, $M / M / 1 / H$ and $M / M / 1 / H$ with catastrophes, where we explicitly know the eigenvalues of $\mathrm{Q}$ by formula rather than having to compute them numerically. Further research to determine which $Q$ matrices have eigenvalues following such pretty patterns would be worth while.

2. Dual processes are proving to be a useful tool for finding transient probability functions in a variety of settings, see: Chang et al.(2004), Green et al.(2003), Krinik et al.(2002), Krinik and Mortensen (2004), Krinik et al.(2004). The main advantage of working with dual processes is that it is often easier to algebraically or combinatorially analyze absorbing Markov processes than recurrent Markov processes.

\section{Acknowledgement}

The authors wish to acknowledge the steady encouragement of Sri Gopal Mohanty who throughout his distinguished career has contributed and promoted the analysis of transient behavior in queueing theory using combinatorial methods. 
Dual Processes To Solve Single Server Systems

\section{References}

Anderson, W. J. (1991). Continuous-Time Markov Chains, An Applications-Oriented Approach. Springer-Verlag, New York.

Bhattacharya, R. N. and E. C. Waymire (1990). Stochastic Processes With Applications. John Wiley \& Sons, New York.

Bohm, W., A. Krinik, and S. G. Mohanty (1997). The Combinatorics of Birth-Death Processes and Applications to Queues. Queueing Systems, 26, 255-267.

Chang, I., A. Krinik, R. J. Swift (2004). Birth-Multiple Catastrophe Processes. (preprint).

Economou, A. and D. Fakinos (2002). A Continuous-time Markov Chain Under the Influence of a Regulating Point Process and Applications in Stochastic Models with Catastrophes. (preprint).

Green, M. L., A, Krinik, C. Mortensen, G. Rubino, R. J. Swift (2003). Transient Probability Functions: A Sample Path Approach, Discrete Mathematics and Theoretical Computer Science, Paris.

Gross, D. and C.M. Harris (1985). Fundamentals of Queueing Theory, Second Edition. John Wiley and Sons, New York.

Kasfy, H. (2004). Dual Processes to Determine Transient Probability Functions. Masters Thesis, California State Polytechnic University, Pomona, California.

Kemperman, J. H. B. (1961). The Passage Problem for a Stationary Markov Chain, Statistical Research Monographs, Volume1. The University of Chicago Press, Illinois.

Krinik, A., C. Mortensen, G. Rubino, (2004). Connections Between Birth-Death Processes. In: A. C. Krinik, Randall J. Swift, Eds., Stochastic Processes and Functional Analysis, A Volume of Recent Advances in Honor of M.M. Rao, Volume 238 in the Lecture Notes in Pure and Applied Mathematical Series, Marcel Dekker, New York, 219-240.

Krinik, A and C. Mortensen (2004). Transient Probability Functions of Finite Birth-Death Processes with Catastrophes. (preprint).

Krinik, A., D. Marcus, D. Kalman and T. Cheng (1997). Transient Solution of the M/M/1 Queueing System via Randomization. In: J. Goldstein, N. Gretsky and J.J. Uhl, Eds., Stochastic Processes and Functional Analysis, Volume 186 in the Lecture Notes in Pure and Applied Mathematical Series, Marcel Dekker, New York, 137-145.

Krinik, A., G. Rubino, R. J. Swift, M. L. Green, H. Lam (2002). Dual Processes to Solve Queueing Systems With Catastrophes.(preprint). 
Dual Processes To Solve Single Server Systems

Kumar, B. K. and D. Arivudainambi (2000). Transient Solution of an M/M/1 Queue with Catastrophes. Computer and Mathematics with Applications, 40, 1233-1240.

Lam H., (2004) Analyzing Single and Two Server Queueing Systems Using Dual Processes. Masters Thesis, California State Polytechnic University, Pomona, California.

Leguesdron, P., J. Pellaumail, G. Rubino, B. Sericola (1993). Transient Solution of the M/M/1 Queue. Adv. Appl. Probability, 25.

Mohanty, S. G. (1979). Lattice Path Counting and Applications, Academic Press, New York. Narayana, T. V. (1979). Lattice Path Combinatorics With Statistical Applications. University of Toronto Press, Toronto.

Swift, R. J. (2000). A Simple Immigration-Catastrophe Process. Math. Scientist, 25, 32-36.

Takacs, L. (1962). Introduction to the Theory of Queues, Oxford University Press, Oxford. 